\title{
Structure of Solutions of Nonlinear Partial Differential Equations of Gérard-Tahara Type
}

\author{
By \\ Hidetoshi TAHARA*广 and Hiroshi YamazawA**
}

\begin{abstract}
Let us consider the following nonlinear singular partial differential equation

$$
\left(t \frac{\partial}{\partial t}\right)^{m} u=F\left(t, x,\left\{\left(t \frac{\partial}{\partial t}\right)^{j}\left(\frac{\partial}{\partial x}\right)^{\alpha} u\right\}_{j+|\alpha| \leq m, j<m}\right)
$$

in the complex domain. When the equation is of Fuchsian type with respect to $t$, holomorphic and singular solutions were investigated quite well by Gérard-Tahara under some assumptions on characteristic exponents. In this paper, the same type of equations is solved in the general case without any assumption on characteristic
\end{abstract} exponents.

\section{$\S 1$. Introduction}

Let $\mathbb{C}$ be the complex plane or the set of all complex numbers, $t$ be the variable in $\mathbb{C}_{t}$, and $x=\left(x_{1}, \ldots, x_{n}\right)$ be the variable in $\mathbb{C}_{x}^{n}=\mathbb{C}_{x_{1}} \times \cdots \times \mathbb{C}_{x_{n}}$. We use the notations: $\mathbb{N}=\{0,1,2, \ldots\}, \mathbb{N}^{*}=\{1,2, \ldots\}, \alpha=\left(\alpha_{1}, \ldots, \alpha_{n}\right) \in$ $\mathbb{N}^{n},|\alpha|=\alpha_{1}+\cdots+\alpha_{n}$, and $(\partial / \partial x)^{\alpha}=\left(\partial / \partial x_{1}\right)^{\alpha_{1}} \cdots\left(\partial / \partial x_{n}\right)^{\alpha_{n}}$. For $\alpha=$ $\left(\alpha_{1}, \ldots, \alpha_{n}\right) \in \mathbb{N}^{n}$ and $\beta=\left(\beta_{1}, \ldots, \beta_{n}\right) \in \mathbb{N}^{n}$ we write $\beta \leq \alpha$ if $\beta_{i} \leq \alpha_{i}$ holds for all $i=1, \ldots, n$.

Communicated by T. Kawai. Received November 19, 2003. Revised February 10, 2004. 2000 Mathematics Subject Classification(s): 35A20, 35G20, 35C10.

† Supported in part by Grant-in-Aid for Scientific Research No. 14540185 of the Japan Society for the Promotion of Science.

*Department of Mathematics, Sophia University, Tokyo 102-8554, Japan.

e-mail: h-tahara@hoffman.cc.sophia.ac.jp

${ }^{* *}$ Department of Language and Culture, Caritas Junior College, Yokohama 225-0011, Japan.

e-mail: yamazawa@caritas.ac.jp

(C) 2005 Research Institute for Mathematical Sciences, Kyoto University. All rights reserved. 
Let $m \in \mathbb{N}^{*}$ be fixed and set

$$
\begin{aligned}
& I_{m}=\left\{(j, \alpha) \in \mathbb{N} \times \mathbb{N}^{n} ; j+|\alpha| \leq m \text { and } j<m\right\}, \\
& \left.N=\# I_{m} \text { (the number of elements of } I_{m}\right), \\
& Z=\left\{Z_{j, \alpha}\right\}_{(j, \alpha) \in I_{m}} \in \mathbb{C}^{N} .
\end{aligned}
$$

Let $F(t, x, Z)$ be a function in the variables $(t, x, Z)$ defined in a neighborhood $\Delta$ of the origin of $\mathbb{C}_{t} \times \mathbb{C}_{x}^{n} \times \mathbb{C}_{Z}^{N}$. Let us consider the equation

$$
\left(t \frac{\partial}{\partial t}\right)^{m} u=F\left(t, x,\left\{\left(t \frac{\partial}{\partial t}\right)^{j}\left(\frac{\partial}{\partial x}\right)^{\alpha} u\right\}_{(j, \alpha) \in I_{m}}\right)
$$

with the unknown function $u=u(t, x)$. Set $\Delta_{0}=\Delta \cap\{t=0, Z=0\}$. Our main assumptions are:

$\left.\mathrm{A}_{1}\right) \quad F(t, x, Z)$ is a holomorphic function on $\Delta$;

$\left.\mathrm{A}_{2}\right) \quad F(0, x, 0) \equiv 0$ on $\Delta_{0}$.

In the study of singularities of solutions of nonlinear partial differential equations of the normal form, the investigation of the above type of equations has become very important (see Kobayashi [9], Lope-Tahara [12] and Tahara [15]). If we set $I_{m}(+)=\left\{(j, \alpha) \in \mathbb{N} \times \mathbb{N}^{n} ; j+|\alpha| \leq m, j<m\right.$ and $\left.|\alpha|>0\right\}$ the situation is divided into the following three cases:

Case $1: \frac{\partial F}{\partial Z_{j, \alpha}}(0, x, 0) \equiv 0$ on $\Delta_{0}$ for all $(j, \alpha) \in I_{m}(+)$;

Case $2: \frac{\partial F}{\partial Z_{j, \alpha}}(0,0,0) \neq 0$ for some $(j, \alpha) \in I_{m}(+)$;

Case 3 : the other cases.

In Case 1, equation (E) is recently called a Gérard-Tahara type partial differential equation (or before it was called a nonlinear Fuchsian type partial differential equation) and it was studied by Gérard-Tahara [5], [6] under some assumptions on characteristic exponents. In Case 2, equation (E) is called a spacially nondegenerate type partial differential equation: Gérard-Tahara [7] discussed a particular class of Case 2 and proved the existence of holomorphic solutions and also singular solutions of (E). In Case 3, equation (E) is called a nonlinear totally characteristic type partial differential equation, and it was studied by Chen-Tahara [2],[3] and Tahara [16].

In this paper we will discuss Case 1 again and determine all the singular solutions of (E) under no assumptions on the characteristic exponents. 


\section{§2. Main Result}

We will consider only Case 1 and so we assume:

$$
\left.\mathrm{A}_{3}\right) \frac{\partial F}{\partial Z_{j, \alpha}}(0, x, 0) \equiv 0 \text { on } \Delta_{0} \text { for all }(j, \alpha) \in I_{m}(+) .
$$

Then, the indicial polynomial $C(\lambda, x)$ of $(\mathrm{E})$ is defined by

$$
C(\lambda, x)=\lambda^{m}-\sum_{j<m} \frac{\partial F}{\partial Z_{j, 0}}(0, x, 0) \lambda^{j}
$$

and the characteristic exponents $\lambda_{1}(x), \ldots, \lambda_{m}(x)$ of $(\mathrm{E})$ are defined by the roots of the equation $C(\lambda, x)=0$ in $\lambda$.

We denote by:

- $\mathcal{R}(\mathbb{C} \backslash\{0\})$ the universal covering space of $\mathbb{C} \backslash\{0\}$,

- $S_{\theta}$ the sector $\{t \in \mathcal{R}(\mathbb{C} \backslash\{0\}) ;|\arg t|<\theta\}$ in $\mathcal{R}(\mathbb{C} \backslash\{0\})$,

- $S(\varepsilon(s))$ the domain $\{t \in \mathcal{R}(\mathbb{C} \backslash\{0\}) ; 0<|t|<\varepsilon(\arg t)\}$, where $\varepsilon(s)$ is a positive-valued continuous function on $\mathbb{R}_{s}$,

- $D_{r}$ the polydisk $\left\{x=\left(x_{1}, \ldots, x_{n}\right) \in \mathbb{C}^{n} ;\left|x_{i}\right|<r\right.$ for $\left.i=1, \cdots, n\right\}$,

- $\mathbb{C}\{x\}$ the ring of convergent power series in $x$, or equivalently, the ring of germs of holomorphic functions at the origin of $\mathbb{C}_{x}^{n}$.

We will determine all the singular solutions of (E) belonging in the class $\widetilde{\mathcal{O}}_{+}$, which is defined by:

Definition 1. We denote by $\widetilde{\mathcal{O}}_{+}$the set of all $u(t, x)$ satisfying the following i) and ii): i) $u(t, x)$ is a holomorphic function on $S(\varepsilon(s)) \times D_{r}$ for some positive-valued continuous function $\varepsilon(s)$ on $\mathbb{R}_{s}$ and $r>0$; and ii) there is an $a>0$ such that for any $0<r_{1}<r$ and $\theta>0$ we have

$$
\max _{x \in D_{r_{1}}}|u(t, x)|=O\left(|t|^{a}\right) \quad\left(\text { as } t \longrightarrow 0 \text { in } S_{\theta}\right) .
$$

Let us first recall the result in Gérard-Tahara [5]. Set

$$
\mu=\#\left\{i \in\{1,2, \ldots, m\} ; \operatorname{Re} \lambda_{i}(0)>0\right\} .
$$

When $\mu=0$, this means that $\operatorname{Re} \lambda_{i}(0) \leq 0$ holds for all $i=1, \ldots, m$. When $\mu \geq 1$, by a renumeration we may assume

$$
\left\{\begin{array}{l}
\operatorname{Re} \lambda_{i}(0)>0 \text { for } 1 \leq i \leq \mu, \\
\operatorname{Re} \lambda_{i}(0) \leq 0 \text { for } \mu+1 \leq i \leq m .
\end{array}\right.
$$


Theorem 1 (Gérard-Tahara (1993)). Assume the conditions $\mathrm{A}_{1}$ ), $\left.\mathrm{A}_{2}\right)$ and $\left.\mathrm{A}_{3}\right)$. Then we have the following results.

(I)(Holomorphic solutions) If $\lambda_{i}(0) \notin \mathbb{N}^{*}$ holds for all $i=1, \ldots, m$, the equation (E) has a unique holomorphic solution $u_{0}(t, x)$ satisfying $u_{0}(0, x) \equiv 0$.

(II)(Singular solutions) Denote by $\mathcal{S}_{+}$the set of all $\widetilde{\mathcal{O}}_{+}$-solutions of $(\mathrm{E})$. Then:

(II-1) When $\mu=0$, we have

$$
\mathcal{S}_{+}=\left\{u_{0}\right\}
$$

where $u_{0}$ is the unique holomorphic solution obtained in (I).

(II-2) When $\mu \geq 1$, under (2.1) and the following additional conditions:

$$
\begin{array}{ll}
\text { c-1) } & \lambda_{i}(0) \neq \lambda_{j}(0) \text { for } 1 \leq i \neq j \leq \mu, \\
\text { c-2) } & C(1,0) \neq 0, \\
\text { c-3) } & C\left(i+j_{1} \lambda_{1}(0)+\cdots+j_{\mu} \lambda_{\mu}(0), 0\right) \neq 0 \text { for any }(i, j) \in \mathbb{N} \times \mathbb{N}^{\mu} \\
& \text { satisfying } i+|j| \geq 2,
\end{array}
$$

we have

$$
\mathcal{S}_{+}=\left\{U\left(\varphi_{1}, \ldots, \varphi_{\mu}\right) ;\left(\varphi_{1}, \ldots, \varphi_{\mu}\right) \in(\mathbb{C}\{x\})^{\mu}\right\},
$$

where $U\left(\varphi_{1}, \ldots, \varphi_{\mu}\right)$ is an $\widetilde{\mathcal{O}}_{+}$-solution of $(\mathrm{E})$ depending on $\left(\varphi_{1}, \ldots, \varphi_{\mu}\right) \in$ $(\mathbb{C}\{x\})^{\mu}$ and having an expansion of the following form:

$$
\begin{aligned}
U\left(\varphi_{1}, \ldots, \varphi_{\mu}\right)= & \sum_{1 \leq p \leq \mu} \varphi_{p}(x) t^{\lambda_{p}(x)}+\sum_{i \geq 1} u_{i}(x) t^{i} \\
& +\sum_{\substack{i+2 m|j| \geq k+2 m \\
|j| \geq 1,(i,|j|) \neq(0,1)}} \phi_{i, j, k}(x) t^{i+j_{1} \lambda_{1}(x)+\cdots+j_{\mu} \lambda_{\mu}(x)}(\log t)^{k} .
\end{aligned}
$$

If one of the conditions c-1) $\sim \mathrm{c}-3$ ) is not satisfied, the expansion of the solution will be much more complicated as is seen in the case $m=1$ by Yamazawa [17], and it seems difficult to describe the expansion in a concrete form. But we can still get the following theorem.

Theorem 2 (Main result). Assume the conditions $\left.\left.\left.\mathrm{A}_{1}\right), \mathrm{A}_{2}\right), \mathrm{A}_{3}\right)$ and $\mu \geq 1$. Denote by $\mathcal{S}_{+}$the set of all $\widetilde{\mathcal{O}}_{+}$-solutions of $(\mathrm{E})$. Then we have

$$
\mathcal{S}_{+}=\left\{U\left(\varphi_{1}, \ldots, \varphi_{\mu}\right) ;\left(\varphi_{1}, \ldots, \varphi_{\mu}\right) \in(\mathbb{C}\{x\})^{\mu}\right\},
$$


where $U\left(\varphi_{1}, \ldots, \varphi_{\mu}\right)$ is an $\widetilde{\mathcal{O}}_{+}$-solution of $(\mathrm{E})$ depending on $\left(\varphi_{1}, \ldots, \varphi_{\mu}\right) \in$ $(\mathbb{C}\{x\})^{\mu}$ and having an expansion of the following form:

$$
U\left(\varphi_{1}, \ldots, \varphi_{\mu}\right)=\sum_{1 \leq p \leq \mu} \varphi_{p}(x) v_{p}(t, x)+\sum_{k \geq 1} \sum_{1 \leq l \leq m_{k}} \phi_{k, l}(x) w_{k, l}(t, x) .
$$

Here, $v_{p}(t, x), m_{k}$ and $w_{k, l}(t, x)$ are as follows: (1) $\left\{v_{1}(t, x), \ldots, v_{\mu}(t, x)\right\}$ is a fundamental system of solutions of $C(t \partial / \partial t, x) v=0$ in $\widetilde{\mathcal{O}}_{+},(2) m_{k}(k \geq 1)$ are positive integers determined by the equation $(\mathrm{E})$, and $(3) w_{k, l}(t, x)(k \geq 1$ and $\left.1 \leq l \leq m_{k}\right)$ are functions also determined by the equation (E) satisfying the following property: there is a $\sigma>0$ such that $w_{k, l}(t, x)=O\left(t^{\sigma k}, \widetilde{\mathcal{O}}_{+}\right)$(as $t \longrightarrow 0)$ holds for all $(k, l)$. The coefficients $\varphi_{p}(x)$ and $\phi_{k, l}(x)$ are as follows: $(4)$ $\varphi_{p}(x)(1 \leq p \leq \mu)$ are arbitrary holomorphic functions, and $(5) \phi_{k, l}(x)(k \geq 1$ and $\left.1 \leq l \leq m_{k}\right)$ are holomorphic functions determined by $\left(\varphi_{1}(x), \ldots, \varphi_{\mu}(x)\right)$.

In the above condition (3) the notation

$$
w(t, x)=O\left(t^{s}, \widetilde{\mathcal{O}}_{+}\right) \quad(\text { as } t \longrightarrow 0)
$$

means that the condition $t^{-s} w(t, x) \in \widetilde{\mathcal{O}}_{+}$holds.

Remark 1. If the condition c-1) is satisfied, we can choose $\left\{v_{1}(t, x), \ldots\right.$, $\left.v_{\mu}(t, x)\right\}$ as $v_{p}(t, x)=t^{\lambda_{p}(x)}$ for $p=1, \ldots, \mu$.

For $m=1$, Theorem 1 was by Gérard-Tahara [4] and the general case as in Theorem 2 was by Yamazawa [17]. In this paper we will prove Theorem 2 in the general case for $m \geq 1$ by a method a little bit different from [17].

Note that our equation (E) is expanded into the form

$$
C\left(t \frac{\partial}{\partial t}, x\right) u=b(x) t+\sum_{p+|q| \geq 2} b_{p, q}(x) t^{p} \prod_{(j, \alpha) \in I_{m}}\left[\left(t \frac{\partial}{\partial t}\right)^{j}\left(\frac{\partial}{\partial x}\right)^{\alpha} u\right]^{q_{j, \alpha}}
$$

where $b(x)$ and $b_{p, q}(x)(p+|q| \geq 2)$ are holomorphic functions in a common neighborhood $\Delta_{0}$ of the origin of $\mathbb{C}_{x}^{n}$, and $p \in \mathbb{N}, q=\left\{q_{j, \alpha}\right\}_{(j, \alpha) \in I_{m}} \in \mathbb{N}^{N}$, and $|q|=\sum_{(j, \alpha) \in I_{m}} q_{j, \alpha}$.

The rest part of this paper is organized as follows. In the next section 3 we will define the system of functions $\left\{w_{k, l}(t, x) ; k \geq 1\right.$ and $\left.1 \leq l \leq m_{k}\right\}$ on which our formal solution (2.4) is based. The properties of these functions $w_{k, l}(t, x)$ will be investigated in Sections 4 and 5 . After these preparations, we will construct a formal solution (2.4) in section 6 , and prove the convergence of this formal solution in Section 7: up to this step we have a family of $\widetilde{\mathcal{O}}_{+^{-}}$ solutions $U\left(\varphi_{1}, \ldots, \varphi_{\mu}\right)$ of $(\mathrm{E})$ and

$$
\mathcal{S}_{+} \supset\left\{U\left(\varphi_{1}, \ldots, \varphi_{\mu}\right) ;\left(\varphi_{1}, \ldots, \varphi_{\mu}\right) \in(\mathbb{C}\{x\})^{\mu}\right\} .
$$


In the last Section 8 we will prove the equality (2.3); that is, we will prove that every $\widetilde{\mathcal{O}}_{+}$-solution $u(t, x)$ of $(\mathrm{E})$ is expressed in the form $u(t, x)=U\left(\varphi_{1}, \ldots, \varphi_{\mu}\right)$ for some $\left(\varphi_{1}, \ldots, \varphi_{\mu}\right) \in(\mathbb{C}\{x\})^{\mu}$.

\section{§3. Definition of the System $\left\{w_{k, l}\right\}$}

Assume the conditions $\left.\left.A_{1}\right), A_{2}\right), A_{3}$ ) and $\mu \geq 1$. Without loss of generality we may assume:

$$
\begin{aligned}
\operatorname{Re} \lambda_{0,1}(0) \leq & \cdots \leq \operatorname{Re} \lambda_{0, \mu_{0}}(0) \leq 0<\operatorname{Re} \lambda_{1,1}(0)=\cdots=\operatorname{Re} \lambda_{1, \mu_{1}}(0) \\
& <\operatorname{Re} \lambda_{2,1}(0)=\cdots=\operatorname{Re} \lambda_{2, \mu_{2}}(0) \\
& <\cdots<\operatorname{Re} \lambda_{d, 1}(0)=\cdots=\operatorname{Re} \lambda_{d, \mu_{d}}(0)
\end{aligned}
$$

where $\mu_{0}=m-\mu, \mu_{i} \geq 1(i=1, \ldots, d)$ and $\mu_{1}+\cdots+\mu_{d}=\mu$. Set

$$
a_{i}=\operatorname{Re} \lambda_{i, 1}(0)=\cdots=\operatorname{Re} \lambda_{i, \mu_{i}}(0), \quad i=1, \ldots, d .
$$

We have $0<a_{1}<a_{2}<\cdots<a_{d}<\infty$. We choose a constant $\sigma$ such that $0<$ $\sigma<\min \left\{1, a_{1}, a_{2}-a_{1}, \ldots, a_{d}-a_{d-1}\right\}$ and $\{\sigma k ; k=1,2, \ldots\} \cap\left\{a_{1}, a_{2}, \ldots, a_{d}\right\}=$ $\varnothing$. Then we have integers $N_{i}(i=1, \ldots, d)$ such that $1 \leq N_{1}<N_{2}<\cdots<$ $N_{d}<\infty$ and that

$$
\sigma N_{i}<a_{i}<\sigma\left(N_{i}+1\right), \quad i=1, \ldots, d
$$

It is easy to see that for $i=0,1, \ldots, d$

$$
C_{i}(\lambda, x)=\left(\lambda-\lambda_{i, 1}(x)\right) \cdots\left(\lambda-\lambda_{i, \mu_{i}}(x)\right)
$$

is a polynomial of degree $\mu_{i}$ in $\lambda$ with coefficients being holomorphic in a neighborhood of $x=0 \in \mathbb{C}_{x}^{n}$. We have a holomorphic decomposition

$$
C(\lambda, x)=C_{0}(\lambda, x) C_{1}(\lambda, x) \cdots C_{d}(\lambda, x)
$$

Let $i \in\{1, \ldots, d\}$. Since the equation

$$
C_{i}\left(t \frac{\partial}{\partial t}, x\right) v=0 \quad \text { in } \widetilde{\mathcal{O}}_{+}
$$

is an ordinary differential equation in $t$ of Euler type with a holomorphic parameter $x$ and since $t=0$ is a regular singular point, we have a fundamental 
system $\left\{v_{i, 1}(t, x), \ldots, v_{i, \mu_{i}}(t, x)\right\}$ of solutions of $(3.6)_{i}$ in the following sense:

v-1) $v_{i, j}(t, x)$ is an $\widetilde{\mathcal{O}}_{+}$-solution of $(3.6)_{i}$;

$\mathrm{v}-2)$ if $v(t, x)$ is an $\widetilde{\mathcal{O}}_{+}$-solution of $(3.6)_{i}, v(t, x)$ is expressed in the form

$$
v(t, x)=\sum_{j=1}^{\mu_{i}} \varphi_{j}(x) v_{i, j}(t, x)
$$

for some unique $\left(\varphi_{1}(x), \ldots, \varphi_{\mu_{i}}(x)\right) \in(\mathbb{C}\{x\})^{\mu_{i}}$.

Moreover, by the conditions (3.2) and (3.3) we have $v_{i, j}(t, x)=O\left(t^{\sigma N_{i}}, \widetilde{\mathcal{O}}_{+}\right)$(as $t \longrightarrow 0)$ for $j=1, \ldots, \mu_{i}$. Since the equation is defined on $\left(\mathbb{C}_{t} \backslash\{0\}\right) \times D_{R}$ for some $R>0$ we see that these $v_{i, j}(t, x)$ 's are holomorphic on $\mathcal{R}\left(\mathbb{C}_{t} \backslash\{0\}\right) \times D_{R}$. For details, see Lemma 1 in Section 4 . We will choose such a fundamental system $\left\{v_{i, 1}(t, x), \ldots, v_{i, \mu_{i}}(t, x)\right\}$ and fix it from now.

For $i=0,1, \ldots, d$ we set

$$
E_{i}\left(\tau_{1}, \ldots, \tau_{\mu_{i}}, x\right)=\frac{1}{\mu_{i} !} \sum_{\pi \in S_{\mu_{i}}}\left(\tau_{1}\right)^{-\lambda_{i, \pi(1)}(x)} \cdots\left(\tau_{\mu_{i}}\right)^{-\lambda_{i, \pi\left(\mu_{i}\right)}(x)}
$$

where $S_{\mu_{i}}$ is the group of permutations of $\left\{1,2, \ldots, \mu_{i}\right\}$. By the theory of symmetric entire functions we see that $E_{i}\left(\tau_{1}, \ldots, \tau_{\mu_{i}}, x\right)$ is a holomorphic function on $(\mathcal{R}(\mathbb{C} \backslash\{0\}))^{\mu_{i}} \times D_{R}$. For a function $f(t, x)$ on $\mathcal{R}\left(\mathbb{C}_{t} \backslash\{0\}\right) \times D_{R}$ we define $\mathcal{R}_{i}[f](t, x)$ and $\mathcal{S}_{i}[f](t, x)$ by the following:

$$
\begin{aligned}
\mathcal{R}_{i}[f](t, x)= & \int_{0}^{t} \frac{d \tau_{\mu_{i}}}{\tau_{\mu_{i}}} \int_{0}^{\tau_{\mu_{i}}} \frac{d \tau_{\mu_{i}-1}}{\tau_{\mu_{i}-1}} \times \\
& \times \cdots \int_{0}^{\tau_{2}} \frac{d \tau_{1}}{\tau_{1}}\left[E_{i}\left(\frac{\tau_{1}}{\tau_{2}}, \ldots, \frac{\tau_{\mu_{i}-1}}{\tau_{\mu_{i}}}, \frac{\tau_{\mu_{i}}}{t}, x\right) f\left(\tau_{1}, x\right)\right], \\
\mathcal{S}_{i}[f](t, x)=( & -1)^{\mu_{i}} \int_{t}^{1} \frac{d \tau_{\mu_{i}}}{\tau_{\mu_{i}}} \int_{\tau_{\mu_{i}}}^{1} \frac{d \tau_{\mu_{i}-1}}{\tau_{\mu_{i}-1}} \times \\
& \times \cdots \int_{\tau_{2}}^{1} \frac{d \tau_{1}}{\tau_{1}}\left[E_{i}\left(\frac{\tau_{1}}{\tau_{2}}, \ldots, \frac{\tau_{\mu_{i}-1}}{\tau_{\mu_{i}}}, \frac{\tau_{\mu_{i}}}{t}, x\right) f\left(\tau_{1}, x\right)\right] .
\end{aligned}
$$

The condition under which the integral (3.7) (and also (3.8)) makes sense will be investigated in Section 4 . If these integrals are well defined, it is easy to see that $\mathcal{R}_{i}[f](t, x)$ (and also $\mathcal{S}_{i}[f](t, x)$ ) gives a solution of the equation

$$
C_{i}\left(t \frac{\partial}{\partial t}, x\right) w=f(t, x)
$$


We define the integral $\mathcal{Q}_{k}[f](t, x)$ by the following:

$$
\mathcal{Q}_{k}[f](t, x)=\left\{\begin{array}{c}
\mathcal{R}_{0} \mathcal{S}_{1} \mathcal{S}_{2} \cdots \mathcal{S}_{d}[f](t, x), \text { if } 1 \leq k \leq N_{1} \\
\mathcal{R}_{0} \mathcal{R}_{1} \mathcal{S}_{2} \cdots \mathcal{S}_{d}[f](t, x), \text { if } N_{1}+1 \leq k \leq N_{2} ; \\
\cdots \cdots \cdots \\
\cdots \cdots \cdots \\
\mathcal{R}_{0} \cdots \mathcal{R}_{d-1} \mathcal{S}_{d}[f](t, x), \text { if } N_{d-1}+1 \leq k \leq N_{d} \\
\mathcal{R}_{0} \cdots \mathcal{R}_{d-1} \mathcal{R}_{d}[f](t, x), \text { if } N_{d}+1 \leq k .
\end{array}\right.
$$

If every integral is well defined, by (3.5) we easily see that $\mathcal{Q}_{k}[f](t, x)$ gives a solution of

$$
C\left(t \frac{\partial}{\partial t}, x\right) w=f(t, x)
$$

By using these integrals, we define:

Definition 2. We define finite sets $\mathcal{F}_{k}(k=1,2, \ldots), \mathcal{G}_{k}(k=1,2, \ldots)$ and $\mathcal{H}_{k}(k=1,2, \ldots)$ of holomorphic functions on $\mathcal{R}\left(\mathbb{C}_{t} \backslash\{0\}\right) \times D_{R}$ inductively by the following procedure $(1) \sim(3)$ :

(1) We set $\mathcal{F}_{1}=\left\{\mathcal{Q}_{1}[t]\right\}$. If $k \geq 2$ and if $\mathcal{H}_{1}, \ldots, \mathcal{H}_{k-1}$ are already defined, we set

$$
\mathcal{F}_{k}=\bigcup_{\substack{2 \leq p+|q| \leq k \\
p \in \mathbb{N}, q \in \mathbb{N}^{N}}} \bigcup_{\begin{array}{r}
k_{1}+\cdots+k_{|q|} \\
=k-p \\
k_{1}, \ldots, k_{|q|} \in \mathbb{N}^{*}
\end{array}}\left\{\mathcal{Q}_{k}\left[t^{p} \psi_{k_{1}} \cdots \psi_{k_{|q|}}\right] ; \psi_{k_{\nu}} \in \mathcal{H}_{k_{\nu}}(\nu=1, \ldots,|q|)\right\},
$$

where $N=\# I_{m}$. If $|q|=0$ we have $p=k$; in this case $\mathcal{Q}_{k}\left[t^{p} \psi_{k_{1}} \cdots \psi_{k_{|q|}}\right]$ should be read as $\mathcal{Q}_{k}\left[t^{k}\right]$. Note that in the right hand side we have $1 \leq k_{\nu} \leq k-1$ for $\nu=1, \ldots,|q|$ and therefore $\mathcal{F}_{k}$ is well defined by the above formula.

(2) If $\mathcal{F}_{k}$ is already defined, we set

$$
\mathcal{G}_{k}= \begin{cases}\mathcal{F}_{k}, & \text { if } k \neq N_{1}, \ldots, N_{d} \\ \mathcal{F}_{k} \cup\left\{v_{i, 1}, \ldots, v_{i, \mu_{i}}\right\} & \text { if } k=N_{i} .\end{cases}
$$

(3) If $\mathcal{G}_{k}$ is already defined, we set

$$
\mathcal{H}_{k}=\bigcup_{(j, \alpha) \in I_{m}} \bigcup_{0 \leq \beta_{j, \alpha} \leq \alpha}\left\{k^{\left|\beta_{j, \alpha}\right|}\left(t \frac{\partial}{\partial t}\right)^{j}\left(\frac{\partial}{\partial x}\right)^{\alpha-\beta_{j, \alpha}} W ; W \in \mathcal{G}_{k}\right\} .
$$

Definition 3. We define the system of functions $\left\{w_{k, l}(t, x) ; k \geq 1,1 \leq\right.$ $\left.l \leq m_{k}\right\}$ by the following: set $m_{k}=\# \mathcal{F}_{k}$ (the number of elements of the set 
$\left.\mathcal{F}_{k}\right)$ and

$$
\mathcal{F}_{k}=\left\{w_{k, 1}(t, x), \ldots, w_{k, m_{k}}(t, x)\right\} \quad \text { for } k=1,2, \ldots
$$

It is clear that $m_{1}=1$ and $\mathcal{F}_{1}=\left\{w_{1,1}(t, x)\right\}$ with $w_{1,1}=\mathcal{Q}_{1}[t]$.

Remark 2. (1) The sets $\mathcal{H}_{k}(k \geq 1)$ are introduced only to avoid the confusion of subscripts in (1) of Definition 2.

(2) In the above finite sets $\mathcal{F}_{k}, \mathcal{G}_{k}$ and $\mathcal{H}_{k}$, every two elements with different labels (i.e., $p, q, k_{1}, \ldots, k_{|q|}$ and so on) are regarded as different elements, even if they are the same function. Hence, if we set $M_{k}=\# \mathcal{G}_{k}, J_{m}=\{(j, \alpha, \beta) \in$ $\left.\mathbb{N} \times \mathbb{N}^{n} \times \mathbb{N}^{n} ;(j, \alpha) \in I_{m}, 0 \leq \beta \leq \alpha\right\}$ and

$$
\begin{aligned}
\mathcal{Z}_{k}=\{ & \left(p, q,\left\{k_{i}\right\}_{i=1}^{|q|},\left\{l_{i}\right\}_{i=1}^{|q|},\left\{\left(j_{i}, \alpha(i), \beta(i)\right)\right\}_{i=1}^{|q|}\right) \in \mathbb{N} \times \mathbb{N}^{N} \times \\
& \times\left(\mathbb{N}^{*}\right)^{|q|} \times\left(\mathbb{N}^{*}\right)^{|q|} \times\left(\mathbb{N} \times \mathbb{N}^{n} \times \mathbb{N}^{n}\right)^{|q|} ; 2 \leq p+|q| \leq k, \\
& p+k_{1}+\cdots+k_{|q|}=k, 1 \leq l_{i} \leq M_{k_{i}}(i=1, \ldots,|q|), \\
& \left.\left(j_{i}, \alpha(i), \beta(i)\right) \in J_{m}(i=1, \ldots,|q|)\right\}
\end{aligned}
$$

we have $m_{k}=\# \mathcal{Z}_{k}$.

The basic properties of these functions are as follows:

Proposition 1. Let $\sigma>0$ be the one in (3.3). If $R>0$ is sufficiently small, we have:

(1) $w_{k, l}(t, x)\left(k \geq 1,1 \leq l \leq m_{k}\right)$ are holomorphic on $\mathcal{R}\left(\mathbb{C}_{t} \backslash\{0\}\right) \times D_{R}$.

(2) For any $\theta>0$ there is a $\delta>0$ such that

$$
\left|\left(t \frac{\partial}{\partial t}\right)^{j}\left(\frac{\partial}{\partial x}\right)^{\alpha} w_{k, l}(t, x)\right| \leq \frac{1}{k^{m-j-|\alpha|}}|t|^{\sigma k} \quad \text { on } S_{\theta}(\delta) \times D_{R}
$$

holds for any $k \geq 1,1 \leq l \leq m_{k}$ and $(j, \alpha) \in I_{m}$, where $S_{\theta}(\delta)=\{t \in$ $\mathcal{R}(\mathbb{C} \backslash\{0\}) ;|\arg t|<\theta$ and $0<|t|<\delta\}$.

In the next Section 4, we will present some preparatory lemmas which are needed in proving Proposition 1; then in Section 5 we will give a proof of this proposition.

\section{$\S 4$. Some Lemmas}

We will present some preparatory lemmas for the proof of Proposition 1 . In this section we use the following notation: 
Definition 4. Let $R>0$ and $s \in \mathbb{R}$.

(1) Let $\theta>0$ and $\delta>0$. We denote by $\mathcal{O}_{s}\left(S_{\theta}(\delta) \times D_{R}\right)$ the set of all holomorphic functions $f(t, x)$ on $S_{\theta}(\delta) \times D_{R}$ satisfying

$$
|f(t, x)| \leq C|t|^{s} \quad \text { on } \quad S_{\theta}(\delta) \times D_{R}
$$

for some $C>0$.

(2) We denote by $\widetilde{\mathcal{O}}_{s}\left(\left(\mathbb{C}_{t} \backslash\{0\}\right) \times D_{R}\right)$ the set of all functions $f(t, x)$ which satisfies the following i) and ii): i) $f(t, x)$ is a holomorphic function on $\mathcal{R}\left(\mathbb{C}_{t} \backslash\{0\}\right) \times D_{R}$, and ii) for any $\theta>0$ and any $\delta>0$ there is a $C>0$ such that $|f(t, x)| \leq C|t|^{s}$ holds on $S_{\theta}(\delta) \times D_{R}$.

Let $p \in \mathbb{N}^{*}$, let $a_{i}(x)(i=1, \ldots, p)$ be bounded holomorphic functions on $D_{R}$, let

$$
P(\xi, x)=\xi^{p}+a_{1}(x) \xi^{p-1}+\cdots+a_{p-1}(x) \xi+a_{p}(x),
$$

denote by $\xi_{1}(x), \ldots, \xi_{p}(x)$ the roots of $P(\xi, x)=0$ in $\xi$, and let us consider the following Euler type homogeneous equation:

$$
P\left(t \frac{\partial}{\partial t}, x\right) v=0
$$

with the unknown function $v(t, x)$. By the theory of ordinary differential equations (or by Proposition 6.3 of Mandai [10]) we know:

Lemma 1. Set

$$
v_{k}(t, x)=\frac{1}{2 \pi \sqrt{-1}} \int_{\Gamma} \frac{\xi^{p-k}}{P(\xi, x)} t^{\xi} d \xi, \quad k=1, \ldots, p,
$$

where $\Gamma$ is a simple closed curve in the complex plane which encloses the set $\left\{\xi_{i}(x) ; i=1, \ldots, p\right.$ and $\left.x \in D_{R}\right\}$. Then we have the following results.

(1) $v_{k}(t, x) \in \widetilde{\mathcal{O}}_{s}\left(\left(\mathbb{C}_{t} \backslash\{0\}\right) \times D_{R}\right)(k=1, \ldots, p)$ hold for any s satisfying $s<\inf \left\{\operatorname{Re} \xi_{i}(x) ; i=1, \ldots, p\right.$ and $\left.x \in D_{R}\right\}$.

(2) If $\varphi_{1}(x), \ldots, \varphi_{p}(x) \in \mathbb{C}\{x\}$ satisfy

$$
\sum_{k=1}^{p} \varphi_{k}(x) v_{k}(t, x)=O\left(t^{b}, \widetilde{\mathcal{O}}_{+}\right)(\text {as } t \longrightarrow 0)
$$

for some $b$ with $b>\max \left\{\operatorname{Re} \xi_{i}(0) ; i=1, \ldots, p\right\}$, then we have $\varphi_{k}(x)=0$ in $\mathbb{C}\{x\}$ for $k=1, \ldots, p$.

(3) If $\operatorname{Re} \xi_{i}(0)>0$ holds for all $i=1, \ldots, p$, the system $\left\{v_{1}(t, x), \ldots\right.$, $\left.v_{p}(t, x)\right\}$ is a fundamental system of $\widetilde{\mathcal{O}}_{+}$-solutions of $(4.2)$. 
Next, let us consider

$$
P\left(t \frac{\partial}{\partial t}, x\right) u=f(t, x) \quad \text { in } \mathcal{O}_{s}\left(S_{\theta}(\delta) \times D_{R}\right) .
$$

Set

$$
E\left(\tau_{1}, \ldots, \tau_{p}, x\right)=\frac{1}{p !} \sum_{\pi \in S_{p}}\left(\tau_{1}\right)^{-\xi_{\pi(1)}(x)} \cdots\left(\tau_{p}\right)^{-\xi_{\pi(p)}(x)}
$$

The following result is due to Baouendi-Goulaouic [1]:

Lemma 2. Let $s \in \mathbb{R}$ and $L>0$. Assume that

$$
s-\operatorname{Re} \xi_{i}(x) \geq L \quad \text { on } D_{R} \quad \text { for } i=1, \ldots, p \text {. }
$$

Then, we have:

(1) For any $f(t, x) \in \mathcal{O}_{s}\left(S_{\theta}(\delta) \times D_{R}\right)$ the equation (4.3) has a unique solution $u(t, x) \in \mathcal{O}_{s}\left(S_{\theta}(\delta) \times D_{R}\right)$ and it is represented by the following integral formula:

$$
u(t, x)=\int_{0}^{t} \frac{d \tau_{p}}{\tau_{p}} \int_{0}^{\tau_{p}} \frac{d \tau_{p-1}}{\tau_{p-1}} \cdots \int_{0}^{\tau_{2}} \frac{d \tau_{1}}{\tau_{1}}\left[E\left(\frac{\tau_{1}}{\tau_{2}}, \ldots, \frac{\tau_{p-1}}{\tau_{p}}, \frac{\tau_{p}}{t}, x\right) f\left(\tau_{1}, x\right)\right] .
$$

(2) If $f(t, x)$ satisfies the estimate (4.1), the solution $u(t, x)$ satisfies

$$
\left|\left(t \frac{\partial}{\partial t}\right)^{j} u(t, x)\right| \leq \frac{A^{j}}{L^{p-j}} C|t|^{s} \quad \text { on } S_{\theta}(\delta) \times D_{R} \quad \text { for } j=0,1, \ldots, p
$$

for any constant $A>0$ with

$$
A \geq \max _{1 \leq i \leq p}\left[1+\frac{\sup _{x \in D_{R}}\left|\xi_{i}(x)\right|}{L}\right] .
$$

Proof. Since the equation (4.3) is written as

$$
\left(t \frac{\partial}{\partial t}-\xi_{1}(x)\right) \cdots\left(t \frac{\partial}{\partial t}-\xi_{p}(x)\right) u=f(t, x)
$$

(1) is obtained by integrating this directly. Let us show (2). Set

$$
u_{1}(t, x)=\int_{0}^{t}\left(\frac{\tau_{1}}{t}\right)^{-\xi_{1}(x)} f\left(\tau_{1}, x\right) \frac{d \tau_{1}}{\tau_{1}}
$$

and

$$
u_{i}(t, x)=\int_{0}^{t}\left(\frac{\tau_{i}}{t}\right)^{-\xi_{i}(x)} u_{i-1}\left(\tau_{i}, x\right) \frac{d \tau_{i}}{\tau_{i}} \text { for } i=2, \ldots, p
$$


Note that $\left(t \partial / \partial t-\xi_{1}(x)\right) u_{1}=f(t, x)$ and $\left(t \partial / \partial t-\xi_{i}(x)\right) u_{i}=u_{i-1}(t, x)(i=$ $2, \ldots, p)$ hold.

Let us first estimate $u_{1}(t, x)$ on $S_{\theta}(\delta) \times D_{R}$. By taking $\left\{\tau_{1} ; \tau_{1}=r t, 0 \leq\right.$ $r \leq 1\}$ as the path of integral we have

$$
\begin{aligned}
\left|u_{1}(t, x)\right| & =\left|\int_{0}^{1} r^{-\xi_{1}(x)-1} f(r t, x) d r\right| \\
& \leq \int_{0}^{1} r^{-\operatorname{Re} \xi_{1}(x)-1} C r^{s}|t|^{s} d r=C|t|^{s} \int_{0}^{1} r^{s-\operatorname{Re} \xi_{1}(x)-1} d r \\
& \leq C|t|^{s} \int_{0}^{1} r^{L-1} d r=C|t|^{s} \frac{1}{L} \quad \text { on } S_{\theta}(\delta) \times D_{R},
\end{aligned}
$$

and by using $(t \partial / \partial t) u_{1}=\xi_{1}(x) u_{1}+f(t, x)$ we see

$$
\begin{aligned}
& \left|\left(t \frac{\partial}{\partial t}\right) u_{1}(t, x)\right|=\left|\xi_{1}(x) u_{1}(t, x)+f(t, x)\right| \\
& \quad \leq\left[\sup _{x \in D_{R}}\left|\xi_{1}(x)\right|\right] C|t|^{s} \frac{1}{L}+C|t|^{s}=\left[\frac{\sup _{x \in D_{R}}\left|\xi_{1}(x)\right|}{L}+1\right] C|t|^{s} \\
& \quad \leq A C|t|^{s} \text { on } S_{\theta}(\delta) \times D_{R} .
\end{aligned}
$$

Let us next estimate $u_{2}(t, x)$ on $S_{\theta}(\delta) \times D_{R}$. Since $\left(t \partial / \partial t-\xi_{2}(x)\right) u_{2}=$ $u_{1}(t, x)$ holds and since $u_{1}(t, x)$ satisfies the estimate $(4.5)$ on $S_{\theta}(\delta) \times D_{R}$, by the same argument as in the case $u_{1}$ we have

$$
\left|u_{2}(t, x)\right| \leq \frac{1}{L^{2}} C|t|^{s} \text { and }\left|\left(t \frac{\partial}{\partial t}\right) u_{2}(t, x)\right| \leq \frac{A}{L} C|t|^{s} \quad \text { on } S_{\theta}(\delta) \times D_{R} .
$$

Moreover, by using $(t \partial / \partial t)^{2} u_{2}=\xi_{2}(x)(t \partial / \partial t) u_{2}+(t \partial / \partial t) u_{1}$ and (4.6) we have

$$
\begin{aligned}
& \left|\left(t \frac{\partial}{\partial t}\right)^{2} u_{2}(t, x)\right| \leq\left|\xi_{2}(x)\right|\left|\left(t \frac{\partial}{\partial t}\right) u_{2}(t, x)\right|+\left|\left(t \frac{\partial}{\partial t}\right) u_{1}(t, x)\right| \\
& \quad \leq\left[\sup _{x \in D_{R}}\left|\xi_{2}(x)\right|\right] \frac{A}{L} C|t|^{s}+A C|t|^{s}=\left[\frac{\sup _{x \in D_{R}}\left|\xi_{2}(x)\right|}{L}+1\right] A C|t|^{s} \\
& \quad \leq A^{2} C|t|^{s} \text { on } S_{\theta}(\delta) \times D_{R} .
\end{aligned}
$$

Thus, by repeating the same argument as above we can obtain the estimate

$$
\left|\left(t \frac{\partial}{\partial t}\right)^{j} u_{p}(t, x)\right| \leq \frac{A^{j}}{L^{p-j}} C|t|^{s} \quad \text { on } S_{\theta}(\delta) \times D_{R} \text { for } j=0,1, \ldots, p .
$$

Since $u_{p}(t, x)=u(t, x)$ holds (by the uniqueness of the solution in (1)), this completes the proof of the part (2). 
Let us also consider the Cauchy problem with initial data on $\{t=\delta\}$ :

$$
\left\{\begin{array}{l}
P\left(t \frac{\partial}{\partial t}, x\right) u=f(t, x) \quad \text { in } \mathcal{O}_{s}\left(S_{\theta}(\delta) \times D_{R}\right), \\
\left.\left(t \frac{\partial}{\partial t}\right)^{j} u\right|_{t=\delta}=0 \quad \text { for } j=0, \ldots, p-1
\end{array}\right.
$$

Lemma 3. Let $s \in \mathbb{R}$ and $L>0$. Assume that

$$
s-\operatorname{Re} \xi_{i}(x) \leq-L \quad \text { on } D_{R} \quad \text { for } i=1, \ldots, p .
$$

Then, we have:

(1) For any $f(t, x) \in \mathcal{O}_{s}\left(S_{\theta}(\delta) \times D_{R}\right)$ the equation (4.7) has a unique solution $u(t, x) \in \mathcal{O}_{s}\left(S_{\theta}(\delta) \times D_{R}\right)$ and it is represented by the following integral formula:

$$
\begin{aligned}
u(t, x)=(-1)^{p} & \int_{t}^{\delta} \frac{d \tau_{p}}{\tau_{p}} \int_{\tau_{p}}^{\delta} \frac{d \tau_{p-1}}{\tau_{p-1}} \times \\
& \times \cdots \int_{\tau_{2}}^{\delta} \frac{d \tau_{1}}{\tau_{1}}\left[E\left(\frac{\tau_{1}}{\tau_{2}}, \ldots, \frac{\tau_{p-1}}{\tau_{p}}, \frac{\tau_{p}}{t}, x\right) f\left(\tau_{1}, x\right)\right] .
\end{aligned}
$$

(2) If $f(t, x)$ satisfies the estimate (4.1) and if $\left|\operatorname{Im} \xi_{i}(x)\right| \leq M(i=1, \ldots, p)$ hold on $D_{R}$, the solution $u(t, x)$ satisfies

$$
\begin{aligned}
& \left|\left(t \frac{\partial}{\partial t}\right)^{j} u(t, x)\right| \leq A^{j}\left(e^{M \theta}\left(\theta+\frac{1}{L}\right)\right)^{p-j} C|t|^{s} \text { on } S_{\theta}(\delta) \times D_{R} \\
& \text { for } j=0,1, \ldots, p
\end{aligned}
$$

for any constant $A>0$ with

$$
A \geq \max _{1 \leq i \leq p}\left[1+\left(e^{M \theta}\left(\theta+\frac{1}{L}\right)\right) \sup _{x \in D_{R}}\left|\xi_{i}(x)\right|\right] .
$$

Proof. (1) is obtained by a direct integration. Let us prove (2). Set

$$
u_{1}(t, x)=-\int_{t}^{\delta}\left(\frac{\tau_{1}}{t}\right)^{-\xi_{1}(x)} f\left(\tau_{1}, x\right) \frac{d \tau_{1}}{\tau_{1}}
$$

and

$$
u_{i}(t, x)=-\int_{t}^{\delta}\left(\frac{\tau_{i}}{t}\right)^{-\xi_{i}(x)} u_{i-1}\left(\tau_{i}, x\right) \frac{d \tau_{i}}{\tau_{i}} \text { for } i=2, \ldots, p .
$$

Note that $\left(t \partial / \partial t-\xi_{1}(x)\right) u_{1}=f(t, x)$ and $\left(t \partial / \partial t-\xi_{i}(x)\right) u_{i}=u_{i-1}(t, x)(i=$ $2, \ldots, p)$ hold. 
Let $(t, x) \in S_{\theta}(\delta) \times D_{R}$ and set $t=|t| \exp (\sqrt{-1} \psi)$. Then, by taking the path $\left\{\tau_{1} ; \tau_{1}=|t| \exp (\sqrt{-1} \varphi), 0 \leq \varphi \leq \psi\right\} \cup\left\{\tau_{1} ; \tau_{1}=r,|t| \leq r \leq \delta\right\}$ we have

$$
\begin{aligned}
\left|u_{1}(t, x)\right| \leq\left|\int_{0}^{\psi}\left(\frac{|t| e^{\sqrt{-1} \varphi}}{|t| e^{\sqrt{-1} \psi}}\right)^{-\xi_{1}(x)} f\left(|t| e^{\sqrt{-1} \varphi}, x\right) d \varphi\right| \\
\quad+\left|\int_{|t|}^{\delta}\left(\frac{r}{|t| e^{\sqrt{-1} \psi}}\right)^{-\xi_{1}(x)} f(r, x) \frac{d r}{r}\right| \\
\leq \int_{0}^{\theta} e^{M \theta} C|t|^{s} d \varphi+\int_{|t|}^{\delta} e^{M \theta}\left(\frac{r}{|t|}\right)^{-s-L} C r^{s} \frac{d r}{r} \\
=e^{M \theta} C|t|^{s} \theta+e^{M \theta} C|t|^{s+L} \frac{|t|^{-L}-\delta^{-L}}{L} \\
\leq C|t|^{s}\left(e^{M \theta}\left(\theta+\frac{1}{L}\right)\right)
\end{aligned}
$$

and by using $(t \partial / \partial t) u_{1}=\xi_{1}(x) u_{1}+f(t, x)$ we see

$$
\begin{aligned}
\left|\left(t \frac{\partial}{\partial t}\right) u_{1}(t, x)\right| & =\left|\xi_{1}(x) u_{1}(t, x)+f(t, x)\right| \\
& \leq\left[\sup _{x \in D_{R}}\left|\xi_{1}(x)\right|\right]\left(e^{M \theta}\left(\theta+\frac{1}{L}\right)\right) C|t|^{s}+C|t|^{s} \\
& \leq A C|t|^{s} \text { on } S_{\theta}(\delta) \times D_{R} .
\end{aligned}
$$

Thus, by the same argument as in the proof of Lemma 2 we obtain

$$
\left|\left(t \frac{\partial}{\partial t}\right)^{j} u_{p}(t, x)\right| \leq A^{j}\left(e^{M \theta}\left(\theta+\frac{1}{L}\right)\right)^{p-j} C|t|^{s} \quad \text { on } S_{\theta}(\delta) \times D_{R}
$$

for $j=0,1, \ldots, p$.

This completes the proof of the part (2).

The following result is also very important in the asymptotic analysis (as $t \longrightarrow 0)$ :

Lemma 4. Let $f(t, x) \in \widetilde{\mathcal{O}}_{+}$and let us consider

$$
P\left(t \frac{\partial}{\partial t}, x\right) u=f(t, x) \quad \text { in } \widetilde{\mathcal{O}}_{+} .
$$

(1) If $f(t, x)=O\left(t^{s}, \widetilde{\mathcal{O}}_{+}\right)($as $t \longrightarrow 0)$ for some $s>0$ and if $s>$ $\max \left\{\operatorname{Re} \xi_{i}(0) ; i=1, \ldots, p\right\}$ holds, the equation (4.9) has a unique solution $u(t, x) \in \widetilde{\mathcal{O}}_{+}$satisfying $u(t, x)=O\left(t^{s}, \widetilde{\mathcal{O}}_{+}\right)($as $t \longrightarrow 0)$. 
(2) If $u(t, x) \in \widetilde{\mathcal{O}}_{+}$is a solution of (4.9) and if the following conditions

i) $u(t, x)=O\left(t^{s}, \widetilde{\mathcal{O}}_{+}\right)($as $t \longrightarrow 0)$,

ii) $f(t, x)=O\left(t^{b}, \widetilde{\mathcal{O}}_{+}\right)($as $t \longrightarrow 0)$, and

iii) $b>s>\max \left\{\operatorname{Re} \xi_{i}(0) ; i=1, \ldots, p\right\}$,

then we have $u(t, x)=O\left(t^{b}, \widetilde{\mathcal{O}}_{+}\right)($as $t \longrightarrow 0)$.

(3) If $f(t, x)=O\left(t^{s}, \widetilde{\mathcal{O}}_{+}\right)($as $t \longrightarrow 0)$ for some $s>0$ and if $s<$ $\min \left\{\operatorname{Re} \xi_{i}(0) ; i=1, \ldots, p\right\}$, every solution $u(t, x) \in \widetilde{\mathcal{O}}_{+}$of the equation (4.9) satisfies $u(t, x)=O\left(t^{s}, \widetilde{\mathcal{O}}_{+}\right)($as $t \longrightarrow 0)$.

Proof. (1) is almost the same as Lemma 2. The proof of (2) is as follows. By (1) we have a solution $w(t, x) \in \widetilde{\mathcal{O}}_{+}$of $(4.9)$ satisfying $w(t, x)=O\left(t^{b}, \widetilde{\mathcal{O}}_{+}\right)$ (as $t \longrightarrow 0)$. Then, $P(t \partial / \partial t, x)(u-w)=0$ and $(u-w)(t, x)=O\left(t^{s}, \widetilde{\mathcal{O}}_{+}\right)$(as $t \longrightarrow 0)$. Thus, by the uniqueness part of (1) we obtain $(u-w)(t, x)=0$ and so $u=w=O\left(t^{b}, \widetilde{\mathcal{O}}_{+}\right)$(as $\left.t \longrightarrow 0\right)$.

Let us show (3). Assume that $f(t, x)=O\left(t^{s}, \widetilde{\mathcal{O}}_{+}\right)($as $t \longrightarrow 0)$ and that $0<s<\operatorname{Re} \xi_{i}(0)$ holds for all $i=1, \ldots, p$. By Lemma 3 we know that the equation (4.9) has a solution $w(t, x) \in \widetilde{\mathcal{O}}_{+}$satisfying $w(t, x)=O\left(t^{s}, \widetilde{\mathcal{O}}_{+}\right)$(as $t \longrightarrow 0)$. Moreover, since (4.9) is an ordinary differential equation with a holomorphic parameter $x$, we know by Lemma 1 that (4.9) with $f(t, x)=$ 0 has a fundamental system $\left\{v_{1}(t, x), \ldots, v_{p}(t, x)\right\}$ of $\widetilde{\mathcal{O}}_{+}$-solutions such that $v_{i}(t, x)=O\left(t^{s}, \widetilde{\mathcal{O}}_{+}\right)($as $t \longrightarrow 0)$.

Now let $u(t, x) \in \widetilde{\mathcal{O}}_{+}$be any solution of (4.9). We have $P(t \partial / \partial t, x)(u-$ $w)=0$ and therefore $(u-w)(t, x)$ is expressed in the form $(u-w)(t, x)=$ $\sum_{i=1}^{p} \phi_{i}(x) v_{i}(t, x)$ for some $\phi_{i}(x) \in \mathbb{C}\{x\}(i=1, \ldots, p)$. This leads us to $u(t, x)=w(t, x)+\sum_{i=1}^{p} \phi_{i}(x) v_{i}(t, x)=O\left(t^{s}, \widetilde{\mathcal{O}}_{+}\right)($as $t \longrightarrow 0)$.

For a function $\phi(x)$ on $D_{r}$ we define the norm $\|\phi\|_{r}$ by

$$
\|\phi\|_{r}=\sup _{x \in D_{r}}|\phi(x)| .
$$

In the proof of Proposition 1, we need also the following Nagumo's lemma (see Nagumo [11] or Lemma 5.1.3 of Hörmander [8]):

Lemma 5. If $\phi(x)$ is a holomorphic function on $D_{R}$ and if

$$
\|\phi\|_{r} \leq \frac{C}{(R-r)^{s}} \quad \text { for any } 0<r<R
$$

holds for some $C \geq 0$ and $s \geq 0$, then we have

$$
\left\|\frac{\partial \phi}{\partial x_{i}}\right\|_{r} \leq \frac{(s+1) e C}{(R-r)^{s+1}} \quad \text { for any } 0<r<R \quad \text { and } i=1, \ldots, n .
$$




\section{$\S 5 . \quad$ Proof of Proposition 1}

Let us return to the situation in Section 3. Let $0<a_{1}<a_{2}<\cdots<a_{d}$ be the ones in (3.2), and let $\sigma>0$ and $1 \leq N_{1}<N_{2}<\cdots<N_{d}$ be the ones in (3.3). Under these fixed constants, we choose now $a>0, R>0, L>0$ and $c>0$ so that the following properties h-1) h-4) hold:

h-1) $\sigma<a<\min \left\{1, a_{1}\right\}$;

h-2) $a N_{i}<a_{i}<a\left(N_{i}+1\right)$ for $i=1, \ldots, d$;

h-3) for $k=1,2, \ldots, N_{d}$ we have

$$
\left|a k-\operatorname{Re} \lambda_{i, p}(x)\right| \geq L \quad \text { on } D_{R} \text { for all }(i, p) ;
$$

h-4) for any $k \geq N_{d}+1$ we have

$$
a k-\operatorname{Re} \lambda_{i, p}(x) \geq c k \quad \text { on } D_{R} \text { for all }(i, p) .
$$

Note that this is possible by choosing $a$ sufficiently close to $\sigma$ and by choosing $R, L$ and $c$ sufficiently small. Without loss of generality, we may assume that $0<R \leq 1$ holds and so $1 /(R-r) \geq 1$ holds for any $r \in(0, R)$.

Let $\mathcal{Q}_{k}$ be the operator defined by (3.10). By Lemmas 2 and 3 we have:

Lemma 6. For any $k=1,2, \ldots$ we have the following properties $(1)_{k}$ and $(2)_{k}$, in which the constant $A_{\theta}>0$ is independent of $k, f(t, x), r$ and $j$.

$(1)_{k} \quad$ If $f(t, x) \in \widetilde{\mathcal{O}}_{a k}\left(\left(\mathbb{C}_{t} \backslash\{0\}\right) \times D_{R}\right)$, we have $\mathcal{Q}_{k}[f](t, x) \in \widetilde{\mathcal{O}}_{a k}\left(\left(\mathbb{C}_{t} \backslash\right.\right.$ $\left.\{0\}) \times D_{R}\right)$ and $C(t \partial / \partial t, x) \mathcal{Q}_{k}[f]=f$ on $\mathcal{R}\left(\mathbb{C}_{t} \backslash\{0\}\right) \times D_{R}$.

$(2)_{k} \quad$ Moreover, if $f(t, x)$ satisfies

$$
\|f(t)\|_{r} \leq C|t|^{a k} \quad \text { on } S_{\theta}(1)
$$

for some $0<r<R$ and $\theta>0$, we have the estimate

$$
\left\|\left(t \frac{\partial}{\partial t}\right)^{j} \mathcal{Q}_{k}[f](t)\right\|_{r} \leq \frac{A_{\theta}}{k^{m-j}} C|t|^{a k} \quad \text { on } S_{\theta}(1) \text { for } j=0,1, \ldots, m-1
$$

Proof. Since h-3) and h-4) hold, by applying Lemmas 2 and $3(d+1)$ times we easily see the property $(1)_{k}$. Moreover, if $k=1, \ldots, N_{d}$ and if $f(t, x)$ satisfies the estimate (5.1) we have

$$
\left\|\left(t \frac{\partial}{\partial t}\right)^{j} \mathcal{Q}_{k}[f](t)\right\|_{r} \leq A_{k, j} C|t|^{a k} \quad \text { on } S_{\theta}(1) \text { for } j=0,1, \ldots, m-1
$$

where the constants $A_{k, j}>0\left(1 \leq k \leq N_{d}\right.$ and $\left.0 \leq j \leq m-1\right)$ depend on $\theta$ in general but are independent of $f(t, x)$ and $r$. If $k \geq N_{d}+1$, by (3.10) we have 
$\mathcal{Q}_{k}[f]=\mathcal{R}_{0} \mathcal{R}_{1} \cdots \mathcal{R}_{d}[f] ;$ therefore by h-4) and Lemma 2 we obtain

$$
\left\|\left(t \frac{\partial}{\partial t}\right)^{j} \mathcal{Q}_{k}[f](t)\right\|_{r} \leq \frac{A_{j}}{k^{m-j}} C|t|^{a k} \quad \text { on } S_{\theta}(1) \text { for } j=0,1, \ldots, m-1
$$

where the constants $A_{j}>0(0 \leq j \leq m-1)$ are independent of $\theta, k, f(t, x)$ and $r$.

Thus, by setting

$$
A_{\theta}=\max \left[\max _{\substack{1 \leq k \leq N_{d} \\ 0 \leq j \leq m-1}} A_{k, j} k^{m-j}, \max _{0 \leq j \leq m-1} A_{j}\right]
$$

we obtain the estimate (5.2).

Now, let us give a proof of Proposition 1. Recall that $\left\{v_{i, 1}(t, x), \ldots\right.$, $\left.v_{i, \mu_{i}}(t, x)\right\}$ is a fundamental system of solutions of $(3.6)_{i}$. Since $a N_{i}<\operatorname{Re} \lambda_{i, p}(x)$ holds on $D_{R}$ for $p=1, \ldots, \mu_{i}$, by taking a smaller $R>0$ if necessary we see the following i) and ii): i) $v_{i, h}(t, x) \in \widetilde{\mathcal{O}}_{a N_{i}}\left(\left(\mathbb{C}_{t} \backslash\{0\}\right) \times D_{R}\right)\left(h=1, \ldots, \mu_{i}\right)$, and ii) for any $\theta>0$ there is a $B_{i, \theta}^{*}>0$ such that

$$
\begin{aligned}
& \left\|\left(t \frac{\partial}{\partial t}\right)^{j}\left(\frac{\partial}{\partial x}\right)^{\alpha} v_{i, h}(t)\right\|_{R} \leq B_{i, \theta}^{*}|t|^{a N_{i}} \quad \text { on } S_{\theta}(1) \\
& \text { for any }(j, \alpha) \in I_{m} \text { and } h=1, \ldots, \mu_{i} .
\end{aligned}
$$

Therefore, by using the condition $0<R \leq 1$ and by setting $B_{i, \theta}=B_{i, \theta}^{*} N_{i}^{m}$ we obtain the following estimates:

$$
\left\|\left(t \frac{\partial}{\partial t}\right)^{j}\left(\frac{\partial}{\partial x}\right)^{\alpha} v_{i, h}(t)\right\|_{r} \leq \frac{1}{N_{i}^{m-j-|\alpha|}} \frac{B_{i, \theta}}{(R-r)^{m\left(N_{i}-1\right)}}|t|^{a N_{i}} \text { on } S_{\theta}(1)
$$

for any $0<r<R,(j, \alpha) \in I_{m}$ and $h=1, \ldots, \mu_{i}$.

Recall also that $\mathcal{F}_{1}=\left\{w_{1,1}(t, x)\right\}$ with $w_{1,1}=\mathcal{Q}_{1}[t]$, and that $0<a<1$ holds. Therefore, we may assume that $w_{1,1} \in \widetilde{\mathcal{O}}_{a}\left(\left(\mathbb{C}_{t} \backslash\{0\}\right) \times D_{R}\right)$ and that for any $\theta>0$ there is a $K_{\theta}>0$ which satisfies

$$
\left\|\left(t \frac{\partial}{\partial t}\right)^{j}\left(\frac{\partial}{\partial x}\right)^{\alpha} w_{1,1}(t)\right\|_{R} \leq K_{\theta}|t|^{a} \quad \text { on } S_{\theta}(1) \text { for any }(j, \alpha) \in I_{m} .
$$

By induction on $k$ we have:

Lemma 7. For any $k=1,2, \ldots$ we have the following properties $(1)_{k}$ and $(2)_{k}$, in which the constant $C_{\theta}>0$ is independent of $(j, \alpha), k$ and $l$. 
$(1)_{k} \quad w_{k, l}(t, x) \in \widetilde{\mathcal{O}}_{a k}\left(\left(\mathbb{C}_{t} \backslash\{0\}\right) \times D_{r}\right)$ for any $0<r<R$ and $l=$ $1, \ldots, m_{k}$.

$(2)_{k}$ We have the following estimates for any $\theta>0$ :

$$
\left\|\left(t \frac{\partial}{\partial t}\right)^{j}\left(\frac{\partial}{\partial x}\right)^{\alpha} w_{k, l}(t)\right\|_{r} \leq \frac{1}{k^{m-j-|\alpha|}} \frac{C_{\theta}^{2 k-1}}{(R-r)^{m(k-1)}}|t|^{a k} \quad \text { on } S_{\theta}(1)
$$

for any $0<r<R,(j, \alpha) \in I_{m}$ and $l=1, \ldots, m_{k}$.

Proof. We set

$$
C_{\theta}=\max \left[K_{\theta},\left(B_{1, \theta}\right)^{1 /\left(2 N_{1}-1\right)}, \ldots,\left(B_{d, \theta}\right)^{1 /\left(2 N_{d}-1\right)}, 1,(m e)^{m} A_{\theta}\right],
$$

where $K_{\theta}, B_{i, \theta}$ and $A_{\theta}$ are the constants in (5.5), (5.4) and Lemma 6, respectively. Since $C_{\theta} \geq K_{\theta}$ holds, the case $k=1$ is clear from (5.5). Let us prove the case $k \geq 2$ by induction on $k$.

Suppose that $k \geq 2$ and that $(1)_{i}$ and $(2)_{i}$ are already proved for $i=$ $1, \ldots, k-1$. Then, by the definition of $\mathcal{F}_{k}=\left\{w_{k, 1}(t, x), \ldots, w_{k, m_{k}}(t, x)\right\}$ we see that $w_{k, l}(t, x)$ is expressed in the form

$$
w_{k, l}(t, x)=\mathcal{Q}_{k}\left[t^{p} \psi_{k_{1}} \cdots \psi_{k_{|q|}}\right]
$$

where $\psi_{k_{\nu}} \in \mathcal{H}_{k_{\nu}}(\nu=1, \ldots,|q|), 2 \leq p+|q| \leq k$ and $p+k_{1}+\cdots+k_{|q|}=k$ (by Definitions 2 and 3). Also we know that each $\psi_{k_{\nu}}(t, x)$ is expressed as

$$
\psi_{k_{\nu}}(t, x)=k_{\nu}{ }^{\left|\beta_{j, \alpha}\right|}\left(t \frac{\partial}{\partial t}\right)^{j}\left(\frac{\partial}{\partial x}\right)^{\alpha-\beta_{j, \alpha}} W(t, x)
$$

for some $(j, \alpha) \in I_{m}, 0 \leq \beta_{j, \alpha} \leq \alpha$ and $W(t, x) \in \mathcal{G}_{k_{\nu}}$ (by Definition 2). Since $1 \leq k_{\nu} \leq k-1$ holds, in the case $W(t, x) \in \mathcal{F}_{k_{\nu}}$ by the induction hypothesis we have

$$
\begin{aligned}
\left\|\psi_{k_{\nu}}(t)\right\|_{r} & =k_{\nu}^{\left|\beta_{j, \alpha}\right|}\left\|\left(t \frac{\partial}{\partial t}\right)^{j}\left(\frac{\partial}{\partial x}\right)^{\alpha-\beta_{j, \alpha}} W(t)\right\|_{r} \\
& \leq k_{\nu}^{\left|\beta_{j, \alpha}\right|} \frac{1}{k_{\nu}^{m-j-\left|\alpha-\beta_{j, \alpha}\right|}} \frac{C_{\theta}^{2 k_{\nu}-1}}{(R-r)^{m\left(k_{\nu}-1\right)}}|t|^{a k_{\nu}} \\
& \leq \frac{C_{\theta}{ }^{2 k_{\nu}-1}}{(R-r)^{m\left(k_{\nu}-1\right)}}|t|^{a k_{\nu}} \quad \text { on } S_{\theta}(1) \text { for any } 0<r<R .
\end{aligned}
$$

In the case $k_{\nu}=N_{i}$ and $W(t, x)=v_{i, h}(t, x)$, by using (5.4) and the inequality $B_{i, \theta} \leq C_{\theta}^{2 N_{i}-1}$ we can obtain the same estimate as (5.9). 
Therefore, by the conditions $k_{1}+\cdots+k_{|q|}=k-p, p+|q| \geq 2,0<a<1$, $C_{\theta} \geq 1$ and $1 /(R-r) \geq 1$ we have

$$
\begin{aligned}
\left\|t^{p} \psi_{k_{1}}(t) \cdots \psi_{k_{|q|}}(t)\right\|_{r} & \leq|t|^{p} \frac{C_{\theta}^{2 k_{1}-1}}{(R-r)^{m\left(k_{1}-1\right)}}|t|^{a k_{1}} \cdots \frac{C_{\theta}^{2 k_{|q|}-1}}{(R-r)^{m\left(k_{|q|}-1\right)}}|t|^{a k_{|q|}} \\
& =\frac{C_{\theta}{ }^{2 k-2 p-|q|}}{(R-r)^{m(k-p-|q|)}}|t|^{p+a(k-p)} \\
& \leq \frac{C_{\theta}{ }^{2 k-2}}{(R-r)^{m(k-2)}}|t|^{a k} \quad \text { on } S_{\theta}(1) .
\end{aligned}
$$

Since $\theta>0$ is arbitrary, this implies that $t^{p} \psi_{k_{1}} \cdots \psi_{k_{|q|}} \in \widetilde{\mathcal{O}}_{a k}\left(\left(\mathbb{C}_{t} \backslash\{0\}\right) \times D_{r}\right)$ for any $0<r<R$. Thus, by $(1)_{k}$ of Lemma 6 we see that $w_{k, l}(t, x) \in$ $\widetilde{\mathcal{O}}_{a k}\left(\left(\mathbb{C}_{t} \backslash\{0\}\right) \times D_{r}\right)$ holds for any $0<r<R$.

Moreover, by applying $(2)_{k}$ of Lemma 6 to $(5.8)$ we have

$$
\left\|\left(t \frac{\partial}{\partial t}\right)^{j} w_{k, l}(t)\right\|_{r} \leq \frac{A_{\theta}}{k^{m-j}} \frac{C_{\theta}^{2 k-2}}{(R-r)^{m(k-2)}}|t|^{a k} \quad \text { on } S_{\theta}(1)
$$

for any $0<r<R$ and $j=0,1, \ldots, m-1$

and by using Lemma 5 and $A_{\theta}(m e)^{|\alpha|} \leq A_{\theta}(m e)^{m} \leq C_{\theta}$ we obtain

$$
\begin{aligned}
& \left\|\left(t \frac{\partial}{\partial t}\right)^{j}\left(\frac{\partial}{\partial x}\right)^{\alpha} w_{k, l}(t)\right\|_{r} \\
& \leq \frac{A_{\theta}}{k^{m-j}} \frac{(m(k-2)+1) \cdots(m(k-2)+|\alpha|) e^{|\alpha|} C_{\theta}{ }^{2 k-2}}{(R-r)^{m(k-2)+|\alpha|}}|t|^{a k} \\
& \leq \frac{A_{\theta}}{k^{m-j-|\alpha|}} \frac{(m e)^{|\alpha|} C_{\theta}^{2 k-2}}{(R-r)^{m(k-1)}}|t|^{a k} \\
& \leq \frac{1}{k^{m-j-|\alpha|}} \frac{C_{\theta}^{2 k-1}}{(R-r)^{m(k-1)}}|t|^{a k} \quad \text { on } S_{\theta}(1)
\end{aligned}
$$

for any $0<r<R$ and $(j, \alpha) \in I_{m}$. Thus, we have proved $(2)_{k}$.

Completion of the proof of Proposition 1 . We set $r=R / 2$. Then we see that $w_{k, l}(t, x) \in \widetilde{\mathcal{O}}_{a k}\left(\left(\mathbb{C}_{t} \backslash\{0\}\right) \times D_{R / 2}\right)$ for all $(k, l)$, and that

$$
\begin{aligned}
\left\|\left(t \frac{\partial}{\partial t}\right)^{j}\left(\frac{\partial}{\partial x}\right)^{\alpha} w_{k, l}(t)\right\|_{R / 2} & \leq \frac{1}{k^{m-j-|\alpha|}} \frac{C_{\theta}^{2 k-1}}{(R / 2)^{m(k-1)}}|t|^{a k} \\
& \leq \frac{1}{k^{m-j-|\alpha|}}\left(\frac{C_{\theta}^{2}}{(R / 2)^{m}}|t|^{a-\sigma}\right)^{k}|t|^{\sigma k} \quad \text { on } S_{\theta}(1)
\end{aligned}
$$


holds for any $(j, \alpha) \in I_{m}$ and $(k, l)$. Thus, if we take $\delta>0$ so that

$$
\frac{C_{\theta}^{2}}{(R / 2)^{m}} \delta^{a-\sigma} \leq 1
$$

we obtain the estimate (3.13) with $R$ replaced by $R / 2$. This completes the proof of Proposition 1.

By the proof of Proposition 1, we have:

Corollary to Proposition 1. We have $v_{i, h}(t, x)=O\left(t^{\sigma N_{i}}, \widetilde{\mathcal{O}}_{+}\right)($as $t \longrightarrow 0)$ for all $(i, h)$, and $w_{k, l}(t, x)=O\left(t^{\sigma k}, \widetilde{\mathcal{O}}_{+}\right)($as $t \longrightarrow 0)$ for all $(k, l)$.

\section{$\S 6 . \quad$ Construction of a Formal Solution}

Let us construct a formal solution $u(t, x)$ of the equation (E) in the form

$$
u(t, x)=\sum_{k \geq 1} u_{k}(t, x)
$$

with

$$
u_{k}(t, x)=\left\{\begin{array}{l}
\sum_{l=1}^{m_{k}} \phi_{k, l}(x) w_{k, l}(t, x), \quad \text { if } k \neq N_{1}, \ldots, N_{d}, \\
\sum_{j=1}^{\mu_{i}} \varphi_{i, j}(x) v_{i, j}(t, x)+\sum_{l=1}^{m_{k}} \phi_{k, l}(x) w_{k, l}(t, x), \quad \text { if } k=N_{i},
\end{array}\right.
$$

where $\varphi_{i, j}(x)$ and $\phi_{k, l}(x)$ are suitable holomorphic functions in a common neighborhood of $x=0$. If such a formal solution is constructed, by Corollary to Proposition 1 we have

$$
u_{k}(t, x)=O\left(t^{\sigma k}, \widetilde{\mathcal{O}}_{+}\right) \quad(\text { as } t \longrightarrow 0) \quad \text { for all } k \geq 1 .
$$

Let us decompose our equation (E) under the condition that the solution $u(t, x)$ is expressed in the form (6.1) with the property (6.3). By substituting (6.1) into (2.5) we have formally

$$
\sum_{k \geq 1} C\left(t \frac{\partial}{\partial t}, x\right) u_{k}=b(x) t+\sum_{p+|q| \geq 2} b_{p, q}(x) t^{p} \prod_{(j, \alpha) \in I_{m}}\left[\sum_{k \geq 1} \mathcal{D}_{j, \alpha} u_{k}\right]^{q_{j, \alpha}}
$$

in which we used the following notation

$$
\mathcal{D}_{j, \alpha} u_{k}=\left(t \frac{\partial}{\partial t}\right)^{j}\left(\frac{\partial}{\partial x}\right)^{\alpha} u_{k} .
$$


Therefore by comparing the asymptotic behavior (as $t \longrightarrow 0$ ) of each term in the both sides of (6.4) we have:

$$
C\left(t \frac{\partial}{\partial t}, x\right) u_{1}=b(x) t+O\left(t^{2 \sigma}, \widetilde{\mathcal{O}}_{+}\right) \quad(\text { as } t \longrightarrow 0)
$$

and for $k \geq 2$

$$
\begin{aligned}
\sum_{1 \leq i \leq k} C & \left(t \frac{\partial}{\partial t}, x\right) u_{i} \\
= & b(x) t+\sum_{2 \leq p+|q| \leq k} b_{p, q}(x) t^{p}\left[\sum_{p+|k(q)| \leq k} \prod_{(j, \alpha) \in I_{m}} \prod_{1 \leq i \leq q_{j, \alpha}} \mathcal{D}_{j, \alpha} u_{k_{j, \alpha}(i)}\right] \\
& +O\left(t^{\sigma(k+1)}, \widetilde{\mathcal{O}}_{+}\right) \quad(\operatorname{as} t \stackrel{0}{\longrightarrow},
\end{aligned}
$$

where

$$
\begin{aligned}
k(q) & =\left\{\left(k_{j, \alpha}(i)\right) ;(j, \alpha) \in I_{m}, 1 \leq i \leq q_{j, \alpha}\right\}, \text { and } \\
|k(q)| & =\sum_{(j, \alpha) \in I_{m}}\left(k_{j, \alpha}(1)+\cdots+k_{j, \alpha}\left(q_{j, \alpha}\right)\right) .
\end{aligned}
$$

Thus, from the view point of asymtotic analysis (as $t \longrightarrow 0$ ) the following decomposition will be reasonable:

$$
C\left(t \frac{\partial}{\partial t}, x\right) u_{1}=b(x) t
$$

and for $k \geq 2$

$(6.6)_{k}$

$$
C\left(t \frac{\partial}{\partial t}, x\right) u_{k}=\sum_{2 \leq p+|q| \leq k} b_{p, q}(x) t^{p}\left[\sum_{p+|k(q)|=k} \prod_{(j, \alpha) \in I_{m}} \prod_{1 \leq i \leq q_{j, \alpha}} \mathcal{D}_{j, \alpha} u_{k_{j, \alpha}(i)}\right] .
$$

It should be remarked that in the right hand side of $(6.6)_{k}$ only the terms $u_{1}, \ldots, u_{k-1}$ and their derivatives appear and that $(6.5)$ and $(6.6)_{k}(k=2,3, \ldots)$ give a recurrent family of equations.

Now, we write $W_{k, l}(t, x)=w_{k, l}(t, x)$ for all $(k, l)$, and in the case $k=N_{i}$ we set $W_{k, m_{k}+j}(t, x)=v_{i, j}(t, x)$ for $j=1, \ldots, \mu_{i}$. Also, we set $M_{k}=m_{k}$ if $k \neq N_{1}, \ldots, N_{d}$, and set $M_{k}=m_{k}+\mu_{i}$ if $k=N_{i}$. Then we have

$$
\begin{aligned}
\mathcal{G}_{k} & =\left\{W_{k, l} ; 1 \leq l \leq M_{k}\right\} \\
& =\left\{\begin{array}{l}
\left\{w_{k, l} ; 1 \leq l \leq m_{k}\right\}, \quad \text { if } k \neq N_{1}, \ldots, N_{d}, \\
\left\{w_{k, l} ; 1 \leq l \leq m_{k}\right\} \cup\left\{v_{i, 1}(t, x), \ldots, v_{i, \mu_{i}}(t, x)\right\}, \quad \text { if } k=N_{i},
\end{array}\right.
\end{aligned}
$$


and $u_{k}(t, x)$ in (6.2) is expressed in the form

$$
u_{k}(t, x)=\sum_{l=1}^{M_{k}} \phi_{k, l}(x) W_{k, l}(t, x)
$$

where in the case $k=N_{i}$ we set $\phi_{k, m_{k}+j}(x)=\varphi_{i, j}(t, x)$ for $j=1, \ldots, \mu_{i}$.

Recall that $v_{i, j}(t, x)$ is a homogeneous solution of $C(t \partial / \partial t, x) v=0$ and therefore we have

$$
C\left(t \frac{\partial}{\partial t}, x\right) \sum_{l=1}^{M_{k}} \phi_{k, l}(x) W_{k, l}(t, x)=\sum_{l=1}^{m_{k}} \phi_{k, l}(x)\left[C\left(t \frac{\partial}{\partial t}, x\right) w_{k, l}(t, x)\right]
$$

for all $k \geq 1$. Since $m_{1}=1$, by substituting $(6.8)_{1}$ into (6.5) we have:

$$
\phi_{1,1}(x)\left[C\left(t \frac{\partial}{\partial t}, x\right) w_{1,1}(t, x)\right]=b(x) t .
$$

Since $w_{1,1}(t, x)=\mathcal{Q}_{1}[t]$ we have $C(t \partial / \partial t, x) w_{1,1}=t$ and so $\phi_{1,1}(x)=b(x)$. Thus, we obtain a solution $u_{1}(t, x)$ of the equation (6.5).

Let us suppose $k \geq 2$ and that a solution $u_{i}(t, x)$ of the equation $(6.6)_{i}$ is already obtained in the form $(6.8)_{i}$ for $i=1, \ldots, k-1$. Under these assumptions, let us solve the equation $(6.6)_{k}$ and find a solution $u_{k}(t, x)$ in the form $(6.8)_{k}$. By substituting $(6.8)_{1}, \ldots,(6.8)_{k}$ into the equation $(6.6)_{k}$ we have

$$
\begin{aligned}
& \sum_{l=1}^{m_{k}} \phi_{k, l}(x)\left[C\left(t \frac{\partial}{\partial t}, x\right) w_{k, l}(t, x)\right] \\
& =\sum_{2 \leq p+|q| \leq k} b_{p, q}(x) t^{p}\left[\sum_{p+|k(q)|=k} \sum_{l(q) \in L(q, k(q))} \prod_{(j, \alpha) \in I_{m}} \prod_{1 \leq i \leq q_{j, \alpha}}\{1 \times\right. \\
& \\
& \left.\left.\quad \times \mathcal{D}_{j, \alpha}\left(\phi_{k_{j, \alpha}(i), l_{j, \alpha}(i)}(x) W_{k_{j, \alpha}(i), l_{j, \alpha}(i)}(t, x)\right)\right\}\right],
\end{aligned}
$$

where

$$
\begin{aligned}
& l(q)=\left\{\left(l_{j, \alpha}(i)\right) ;(j, \alpha) \in I_{m} \text { and } 1 \leq i \leq q_{j, \alpha}\right\}, \text { and } \\
& L(q, k(q))=\left\{l(q) ; 1 \leq l_{j, \alpha}(i) \leq M_{k_{j, \alpha}(i)}\right. \\
&\text { holds for all } \left.(j, \alpha) \in I_{m} \text { and } 1 \leq i \leq q_{j, \alpha}\right\} .
\end{aligned}
$$

Hence, if we set

$$
\begin{aligned}
\mathcal{J}_{k}(p, q) & =\{(k(q), l(q)) ; p+|k(q)|=k \text { and } l(q) \in L(q, k(q))\}, \\
\beta(q) & =\left\{\left(\beta_{j, \alpha}(i)\right) ; \beta_{j, \alpha}(i) \in \mathbb{N}^{n},(j, \alpha) \in I_{m} \text { and } 1 \leq i \leq q_{j, \alpha}\right\}, \\
\Gamma(q) & =\left\{\beta(q) ; \beta_{j, \alpha}(i) \leq \alpha \text { holds for all }(j, \alpha) \in I_{m} \text { and } 1 \leq i \leq q_{j, \alpha}\right\},
\end{aligned}
$$


equation $(6.6)_{k}$ is expressed in the form

$$
\begin{aligned}
& \sum_{l=1}^{m_{k}} \phi_{k, l}(x)\left[C\left(t \frac{\partial}{\partial t}, x\right) w_{k, l}(t, x)\right] \\
& =\sum_{2 \leq p+|q| \leq k} b_{p, q}(x) \sum_{(k(q), l(q)) \in \mathcal{J}_{k}(p, q)} \sum_{\beta(q) \in \Gamma(q)} \psi_{k(q), l(q), \beta(q)}(x) \times \\
& \quad \times\left[t^{p} \prod_{(j, \alpha) \in I_{m}} \prod_{1 \leq i \leq q_{j, \alpha}} k_{j, \alpha}(i)^{\left|\beta_{j, \alpha}(i)\right|} \mathcal{D}_{j, \alpha-\beta_{j, \alpha}(i)}\left(W_{k_{j, \alpha}(i), l_{j, \alpha}(i)}(t, x)\right)\right]
\end{aligned}
$$

and $\psi_{k(q), l(q), \beta(q)}(x)$ 's are known functions; precisely they are given by

$$
\begin{aligned}
& \psi_{k(q), l(q), \beta(q)}(x) \\
& =\prod_{(j, \alpha) \in I_{m}} \prod_{1 \leq i \leq q_{j, \alpha}}\left(\begin{array}{c}
\alpha \\
\beta_{j, \alpha}(i)
\end{array}\right) \frac{1}{k_{j, \alpha}(i)^{\left|\beta_{j, \alpha}(i)\right|}}\left(\frac{\partial}{\partial x}\right)^{\beta_{j, \alpha}(i)} \phi_{k_{j, \alpha}(i), l_{j, \alpha}(i)}(x) .
\end{aligned}
$$

We remark again that in the right hand side of (6.9) (and (6.10)) the inequality $1 \leq k_{j, \alpha}(i) \leq k-1$ holds for all $(j, \alpha, i)$ and therefore the right hand side of (6.9) (and (6.10)) can be considered as a known part by the induction hypothesis.

Here we note the following lemma:

Lemma 8. Let $k \geq 2$ and set $\mathcal{A}_{k}=\{(p, q, k(q), l(q), \beta(q)) ; 2 \leq p+$ $|q| \leq k,(k(q), l(q)) \in \mathcal{J}_{k}(p, q)$ and $\left.\beta(q) \in \Gamma(q)\right\}$. Then by a suitable injection $\pi_{k}: \mathcal{A}_{k} \longrightarrow\left\{1,2, \ldots, m_{k}\right\}$ we have the following equality:

$$
\begin{aligned}
& \mathcal{Q}_{k}\left[t^{p} \prod_{(j, \alpha) \in I_{m}} \prod_{1 \leq i \leq q_{j, \alpha}} k_{j, \alpha}(i)^{\left|\beta_{j, \alpha}(i)\right|} \mathcal{D}_{j, \alpha-\beta_{j, \alpha}(i)}\left(W_{k_{j, \alpha}(i), l_{j, \alpha}(i)}(t, x)\right)\right] \\
& =w_{k, l}(t, x)
\end{aligned}
$$

under the correspondence $\pi_{k}(p, q, k(q), l(q), \beta(q))=l$.

Proof. Let $\mathcal{Z}_{k}$ be the set in (3.12). For $q=\left\{q_{j, \alpha}\right\}_{(j, \alpha) \in I_{m}} \in \mathbb{N}^{N}$ we set $S(q)=\left\{(j, \alpha, i) ;(j, \alpha) \in I_{m}\right.$ and $\left.1 \leq i \leq q_{j, \alpha}\right\}$. Then we have $|q|=\# S(q)$. Therefore, by

$$
\mathcal{A}_{k} \ni(p, q, k(q), l(q), \beta(q)) \longmapsto\left(p, q, k(q), l(q),\left\{\left(j, \alpha, \beta_{j, \alpha}(i)\right\}_{(j, \alpha, i) \in S(q)}\right) \in \mathcal{Z}_{k}\right.
$$


we have a natural injection from $\mathcal{A}_{k}$ into $\mathcal{Z}_{k}$. Since $m_{k}=\# \mathcal{Z}_{k}$ (see Remark 2 ), by the definition of $\mathcal{F}_{k}$ we easily obtain this lemma.

Thus, to solve the equation (6.9) it is sufficient to determine the coefficients $\phi_{k, l}(x)$ by

$$
\phi_{k, l}(x)= \begin{cases}0, & \text { if } l \notin \pi_{k}\left(\mathcal{A}_{k}\right), \\ b_{p, q}(x) \psi_{k(q), l(q), \beta(q)}(x), & \text { if } l \in \pi_{k}\left(\mathcal{A}_{k}\right)\end{cases}
$$

under the correspondence $l=\pi_{k}(p, q, k(q), l(q), \beta(q))$.

It is clear that in the case $k=N_{i}$ the coefficients $\varphi_{i, 1}(x), \ldots, \varphi_{i, \mu_{i}}(x)$ of

$$
u_{k}(t, x)=\sum_{j=1}^{\mu_{i}} \varphi_{i, j}(x) v_{i, j}(t, x)+\sum_{l=1}^{m_{k}} \phi_{k, l}(x) w_{k, l}(t, x)
$$

can be chosen arbitrarily.

Thus we have proved.

Proposition 2. We can construct a formal solution $u(t, x)$ of the form (6.1) with (6.2). Moreover we see the following: (i) the coefficients $\varphi_{i, j}(x)$ $\in \mathbb{C}\{x\}\left(1 \leq i \leq d\right.$ and $\left.1 \leq j \leq \mu_{i}\right)$ can be chosen arbitrarily, (ii) $\phi_{1,1}(x)=$ $b(x)$, and (iii) all the other coefficients $\phi_{k, l}(x) \in \mathbb{C}\{x\}$ are determined by (6.12) with (6.10) and therefore they are all holomorphic in a common neighborhood of $x=0 \in \mathbb{C}^{n}$.

\section{$\S 7 . \quad$ Proof of the Convergence of a Formal Solution}

We will prove here the convergence of the formal solution constructed in Proposition 2.

Let a fixed constant $R>0$ be sufficiently small with $R \leq 1$. We take $B \geq 0, B_{p, q} \geq 0(p+|q| \geq 2)$ so that the coefficients $b(x), b_{p, q}(x)(p+|q| \geq 2)$ of (2.5) satisfy

$$
\|b\|_{R} \leq B \quad \text { and } \quad\left\|b_{p, q}\right\|_{R} \leq B_{p, q} \quad(p+|q| \geq 2),
$$


and that the power series

$$
B t+\sum_{p+|q| \geq 2} B_{p, q} t^{p} \prod_{(j, \alpha) \in I_{m}}\left[Z_{j, \alpha}\right]^{q_{j, \alpha}}
$$

is convergent in a neighborhood of $(t, Z)=(0,0) \in \mathbb{C} \times \mathbb{C}^{N}$.

Let

$$
u(t, x)=\sum_{k \geq 1} u_{k}(t, x) \quad \text { with } \quad u_{k}(t, x)=\sum_{l=1}^{M_{k}} \phi_{k, l}(x) W_{k, l}(t, x)
$$

be the formal solution constructed in section 6 and assume that $\phi_{k, l}(x)(k=$ $1,2, \ldots$ and $1 \leq l \leq M_{k}$ ) are all holomorphic on $D_{R}$. By the construction we know that $m_{1}=1$ and $\phi_{1,1}(x)=b(x)$ and that $\phi_{k, l}(x)\left(l=1, \ldots, m_{k}\right)$ for $k \geq 2$ are defined by (6.12). Since $W_{k, l}(t, x)\left(k=1,2, \ldots\right.$ and $\left.1 \leq l \leq M_{k}\right)$ are defined by (6.7), by Proposition 1 and (5.3) we know the following: for any $\theta>0$ there is a $\delta>0$ such that

$$
\left\|W_{k, l}(t)\right\|_{R} \leq|t|^{\sigma k} \quad \text { on } S_{\theta}(\delta) \text { for all }(k, l) .
$$

Therefore, for any $0<r<R$ we have

$$
\left\|u_{k}(t)\right\|_{r} \leq \sum_{l=1}^{M_{k}}\left\|\phi_{k, l}\right\|_{r}\left\|W_{k, l}(t)\right\|_{r} \leq \sum_{l=1}^{M_{k}}\left\|\phi_{k, l}\right\|_{r}|t|^{\sigma k} \quad \text { on } S_{\theta}(\delta) .
$$

Thus, in order to estimate the term $u_{k}(t, x)$ in (7.1) it will be convenient to use the following norm $\left\|u_{k}\right\|_{r}^{*}$ :

$$
\left\|u_{k}\right\|_{r}^{*}=\sum_{l=1}^{M_{k}}\left\|\phi_{k, l}\right\|_{r}
$$

Note that this is expressed also in the form

$$
\left\|u_{k}\right\|_{r}^{*}=\sum_{i=1}^{d} \delta_{k, N_{i}}\left(\sum_{j=1}^{\mu_{i}}\left\|\varphi_{i, j}\right\|_{r}\right)+\sum_{l=1}^{m_{k}}\left\|\phi_{k, l}\right\|_{r}
$$

where $\delta_{k, N_{i}}$ denotes the Kronecker's delta (that is, $\delta_{k, N_{i}}=1$ if $k=N_{i}$, and $\delta_{k, N_{i}}=0$ if $k \neq N_{i}$ ). 
Moreover, in the case $k \geq 2$, by substituting (6.12) (with (6.10)) into (7.4) we have

$$
\begin{aligned}
\left\|u_{k}\right\|_{r}^{*} \leq & \sum_{i=1}^{d} \delta_{k, N_{i}}\left(\sum_{j=1}^{\mu_{i}}\left\|\varphi_{i, j}\right\|_{r}\right)+\sum_{(p, q, k(q), l(q), \beta(q)) \in \mathcal{A}_{k}}\left\|b_{p, q}\right\|_{r}\left\|\psi_{k(q), l(q), \beta(q)}\right\|_{r} \\
\leq & \sum_{i=1}^{d} \delta_{k, N_{i}}\left(\sum_{j=1}^{\mu_{i}}\left\|\varphi_{i, j}\right\|_{r}\right) \\
& +\sum_{(p, q, k(q), l(q), \beta(q)) \in \mathcal{A}_{k}}\left\|b_{p, q}\right\|_{r} \prod_{(j, \alpha) \in I_{m}} \prod_{1 \leq i \leq q_{j, \alpha}}\left(\begin{array}{c}
\alpha \\
\beta_{j, \alpha}(i)
\end{array}\right) \times \\
& \times \frac{1}{k_{j, \alpha}(i)^{\left|\beta_{j, \alpha}(i)\right|}}\left\|\left(\frac{\partial}{\partial x}\right)^{\beta_{j, \alpha}(i)} \phi_{k_{j, \alpha}(i), l_{j, \alpha}(i)}\right\|_{r} ;
\end{aligned}
$$

therefore if we write

$$
\left\|D_{x}^{\beta}\left[u_{h}\right]\right\|_{r}^{*}=\sum_{l=1}^{M_{h}}\left\|\left(\frac{\partial}{\partial x}\right)^{\beta} \phi_{h, l}\right\|_{r}
$$

we obtain

$$
\begin{aligned}
\left\|u_{k}\right\|_{r}^{*} \leq & \sum_{i=1}^{d} \delta_{k, N_{i}}\left(\sum_{j=1}^{\mu_{i}}\left\|\varphi_{i, j}\right\|_{r}\right) \\
& +\sum_{2 \leq p+|q| \leq k} B_{p, q} \sum_{\substack{p+|k(q)|=k \\
\beta(q) \in \Gamma(q)}} \prod_{\substack{(j, \alpha) \in I_{m} \\
1 \leq i \leq q_{j, \alpha}}}\left(\begin{array}{c}
\alpha \\
\beta_{j, \alpha}(i)
\end{array}\right) \times \\
& \times \frac{1}{k_{j, \alpha}(i)^{\left|\beta_{j, \alpha}(i)\right|} \mid}\left\|D_{x}^{\beta_{j, \alpha}(i)}\left[u_{k_{j, \alpha}(i)}\right]\right\|_{r}^{*} .
\end{aligned}
$$

Since $\varphi_{i, j}(x)\left(i=1, \ldots, d\right.$ and $\left.1 \leq j \leq \mu_{i}\right)$ are known holomorphic functions on $D_{R}$, we can find $A_{i}>0(i=1, \ldots, d)$ so that

$$
A_{i} \geq \sum_{j=1}^{\mu_{i}}\left\|\varphi_{i, j}\right\|_{R} \quad(i=1, \ldots, d) .
$$

Now, let us consider the following analytic equation with respect to $Y$ :

$$
\begin{aligned}
Y= & \sum_{i=1}^{d} \frac{A_{i}}{(R-r)^{m\left(2 N_{i}-1\right)}} t^{N_{i}}+\frac{B}{(R-r)^{m}} t \\
& +\frac{1}{(R-r)^{m}} \sum_{p+|q| \geq 2} \frac{B_{p, q}}{(R-r)^{m(2 p+|q|-2)}} t^{p}\left(2^{m}(2 m e)^{m} Y\right)^{|q|}
\end{aligned}
$$

where $r$ is a parameter with $0<r<R$. 
By the implicit function theorem we see that (7.8) has a unique holomorphic solution $Y(t)$ in a neighborhood of $t=0 \in \mathbb{C}$ satisfying $Y(0)=0$, and that the Taylor coefficients $Y_{k}(k=1,2, \ldots)$ of the expansion

$$
Y=\sum_{k \geq 1} Y_{k} t^{k}
$$

satisfy the following recurrence formulas:

$$
Y_{1}=\frac{\delta_{1, N_{1}} A_{1}+B}{(R-r)^{m}}
$$

and for $k \geq 2$

$$
\begin{aligned}
Y_{k}= & \sum_{i=1}^{d} \delta_{k, N_{i}} \frac{A_{i}}{(R-r)^{m\left(2 N_{i}-1\right)}} \\
& +\frac{1}{(R-r)^{m}} \sum_{2 \leq p+|q| \leq k} \frac{B_{p, q}}{(R-r)^{m(2 p+|q|-2)}} \times \\
& \times \sum_{p+|k(q)|=k} \prod_{(j, \alpha) \in I_{m}} \prod_{1 \leq i \leq q_{j, \alpha}}\left(2^{m}(2 m e)^{m} Y_{k_{j, \alpha}(i)}\right) .
\end{aligned}
$$

Moreover, by induction on $k$ we can easily see that $Y_{k}$ has the form

$$
Y_{k}=\frac{C_{k}}{(R-r)^{m(2 k-1)}}, \quad k=1,2, \ldots
$$

where $C_{k} \geq 0(k=1,2, \ldots)$ are constants independent of the parameter $r$.

The following lemma asserts that the Taylor series (7.9) of $Y(t)$ is a majorant series of our formal solution (7.1).

Lemma 9. For any $k=1,2, \ldots$ we have

$$
\frac{1}{k^{|\alpha|}}\left\|D_{x}^{\alpha}\left[u_{k}\right]\right\|_{r}^{*} \leq(2 m e)^{m} Y_{k} \quad \text { for any } 0<r<R \text { and }|\alpha| \leq m \text {. }
$$

Proof. We will prove this by induction on $k$. When $k=1$ we have

$$
u_{1}(t, x)=\delta_{1, N_{1}} \sum_{j=1}^{\mu_{1}} \varphi_{1, j}(x) v_{1, j}(t, x)+b(x) w_{1,1}(t, x) ;
$$


therefore by (7.7), $\|b\|_{R} \leq B$ and by using Lemma 5 we have

$$
\begin{aligned}
\left\|D_{x}^{\alpha}\left[u_{1}\right]\right\|_{r}^{*} & =\delta_{1, N_{1}} \sum_{j=1}^{\mu_{1}}\left\|\left(\frac{\partial}{\partial x}\right)^{\alpha} \varphi_{1, j}\right\|_{r}+\left\|\left(\frac{\partial}{\partial x}\right)^{\alpha} b\right\|_{r} \\
& \leq \delta_{1, N_{1}} \frac{|\alpha| ! e^{|\alpha|} A_{1}}{(R-r)^{|\alpha|}}+\frac{|\alpha| ! e^{|\alpha|} B}{(R-r)^{|\alpha|}}=|\alpha| ! e^{|\alpha|} \frac{\delta_{1, N_{1}} A_{1}+B}{(R-r)^{|\alpha|}} \\
& \leq(2 m e)^{m} \frac{\delta_{1, N_{1}} A_{1}+B}{(R-r)^{m}}=(2 m e)^{m} Y_{1}
\end{aligned}
$$

which proves $(7.13)_{1}$.

Let $k \geq 2$ and suppose that $(7.13)_{i}$ is already proved for all $i=1, \ldots, k-1$.

Then, by (7.6), (7.7) and the induction hypothesis we have

$$
\begin{aligned}
\left\|u_{k}\right\|_{r}^{*} \leq & \sum_{i=1}^{d} \delta_{k, N_{i}} A_{i} \\
& +\sum_{2 \leq p+|q| \leq k} B_{p, q} \sum_{\substack{p+|k(q)|=k \\
\beta(q) \in \Gamma(q)}} \prod_{(j, \alpha) \in I_{m}} \prod_{1 \leq i \leq q_{j, \alpha}}\left(\begin{array}{c}
\alpha \\
\beta_{j, \alpha}(i)
\end{array}\right)(2 m e)^{m} Y_{k_{j, \alpha}(i)} .
\end{aligned}
$$

Hence, if we note that $1 /(R-r)^{m(2 k-2)} \geq 1,1 /(R-r)^{m(2 p+|q|-2)} \geq 1$ and

$$
\sum_{0 \leq \beta \leq \alpha}\left(\begin{array}{l}
\alpha \\
\beta
\end{array}\right)=2^{|\alpha|} \leq 2^{m},
$$

we see

$$
\begin{aligned}
\left\|u_{k}\right\|_{r}^{*} \leq & \sum_{i=1}^{d} \delta_{k, N_{i}} \frac{A_{i}}{(R-r)^{m(2 k-2)}} \\
& +\sum_{2 \leq p+|q| \leq k} \frac{B_{p, q}}{(R-r)^{m(2 p+|q|-2)}} \times \\
& \times \sum_{p+|k(q)|=k} \prod_{(j, \alpha) \in I_{m}} \prod_{1 \leq i \leq q_{j, \alpha}}\left(2^{m}(2 m e)^{m} Y_{k_{j, \alpha}(i)}\right) .
\end{aligned}
$$

Therefore, by comparing (7.11) and (7.14) we obtain

$$
\left\|u_{k}\right\|_{r}^{*} \leq(R-r)^{m} Y_{k}=\frac{C_{k}}{(R-r)^{m(2 k-2)}} \quad \text { for any } 0<r<R .
$$

Thus, by applying Lemma 5 to (7.15) we obtain

$$
\begin{aligned}
\frac{1}{k^{|\alpha|}}\left\|D_{x}^{\alpha}\left[u_{k}\right]\right\|_{r}^{*} & \leq \frac{1}{k^{|\alpha|}} \frac{(m(2 k-2)+1) \cdots(m(2 k-2)+|\alpha|) e^{|\alpha|} C_{k}}{(R-r)^{m(2 k-2)+|\alpha|}} \\
& \leq \frac{(2 m e)^{|\alpha|} C_{k}}{(R-r)^{m(2 k-2)+|\alpha|}} \leq \frac{(2 m e)^{m} C_{k}}{(R-r)^{m(2 k-1)}}=(2 m e)^{m} Y_{k}
\end{aligned}
$$


which proves $(7.13)_{k}$.

Proof of the convergence of the formal solution (7.1). Let $r$ be fixed; for example, we set $r=R / 2$. By (7.3) and (7.13) we have

$$
\sum_{k \geq 1}\left\|u_{k}(t)\right\|_{r} \leq \sum_{k \geq 1}\left\|u_{k}\right\|_{r}^{*}|t|^{\sigma k} \leq(2 m e)^{m} \sum_{k \geq 1} Y_{k}|t|^{\sigma k} \quad \text { on } S_{\theta}(\delta) .
$$

This asserts that the formal solution $u(t, x)$ in $(7.1)$ converges on $S_{\theta}\left(\delta_{1}\right) \times D_{r}$, if $\delta_{1}>0$ is sufficiently small. Since $\theta>0$ is arbitrary, we can conclude that $u(t, x)$ converges in $\widetilde{\mathcal{O}}_{+}$and gives an $\widetilde{\mathcal{O}}_{+}$-solution of $(\mathrm{E})$.

Summing up we have obtained

Theorem 3. The equation (E) has a family of $\widetilde{\mathcal{O}}_{+}$-solutions which is expanded into the form

$$
u(t, x)=\sum_{k \geq 1}\left[\sum_{i=1}^{d} \delta_{k, N_{i}} \sum_{j=1}^{\mu_{i}} \varphi_{i, j}(x) v_{i, j}(t, x)+\sum_{l=1}^{m_{k}} \phi_{k, l}(x) w_{k, l}(t, x)\right],
$$

where (i) the coefficients $\varphi_{i, j}(x) \in \mathbb{C}\{x\}\left(1 \leq i \leq d\right.$ and $\left.1 \leq j \leq \mu_{i}\right)$ can be chosen arbitrarily, (ii) $\phi_{1,1}(x)=b(x)$, and (iii) all the other coefficients $\phi_{k, l}(x) \in \mathbb{C}\{x\}$ are determined by the data $\varphi_{i, j}(x)\left(1 \leq i \leq d\right.$ and $\left.1 \leq j \leq \mu_{i}\right)$ and they are all holomorphic in a common neighborhood of $x=0 \in \mathbb{C}^{n}$.

From now we will write the solution (7.16) as

$$
u(t, x)=U\left(\vec{\varphi}_{1}, \ldots, \vec{\varphi}_{d}\right)
$$

with $\vec{\varphi}_{i}=\left(\varphi_{i, 1}, \ldots, \varphi_{i, \mu_{i}}\right) \in \mathbb{C}\{x\}^{\mu_{i}}$ for $i=1, \ldots, d$. By the construction of $U\left(\vec{\varphi}_{1}, \ldots, \vec{\varphi}_{d}\right)$ and (2) of Lemma 1 we see:

Proposition 3. (1) $U\left(\vec{\varphi}_{1}, \ldots, \vec{\varphi}_{d}\right)=O\left(t^{\sigma}, \widetilde{\mathcal{O}}_{+}\right)($as $t \longrightarrow 0)$.

(2) We have

$$
\begin{aligned}
& U\left(\vec{\varphi}_{1}, \ldots, \vec{\varphi}_{p-1}, \vec{\varphi}_{p}, \overrightarrow{0}, \ldots, \overrightarrow{0}\right)-U\left(\vec{\varphi}_{1}, \ldots, \vec{\varphi}_{p-1}, \overrightarrow{0}, \overrightarrow{0}, \ldots, \overrightarrow{0}\right) \\
& =\sum_{j=1}^{\mu_{p}} \varphi_{p, j}(x) v_{p, j}(t, x)+O\left(t^{\sigma\left(N_{p}+1\right)}, \widetilde{\mathcal{O}}_{+}\right) \quad(\text { as } t \longrightarrow 0)
\end{aligned}
$$

where $\vec{\varphi}_{p}=\left(\varphi_{p, 1}, \ldots, \varphi_{p, \mu_{p}}\right) \in \mathbb{C}\{x\}^{\mu_{p}}$.

(3) If $U\left(\vec{\varphi}_{1}, \ldots, \vec{\varphi}_{d}\right)=U\left(\vec{\psi}_{1}, \ldots, \vec{\psi}_{d}\right)$ we have $\vec{\varphi}_{i}(x)=\vec{\psi}_{i}(x)$ in $\mathbb{C}\{x\}^{\mu_{i}}$ for $i=1, \ldots, d$. 


\section{$\S 8$. Completion of the Proof of Theorem 2}

Denote by $\mathcal{S}_{+}$the set of all $\widetilde{\mathcal{O}}_{+}$-solutions of $(\mathrm{E})$. We already proved that

$$
\mathcal{S}_{+} \supset\left\{U\left(\vec{\varphi}_{1}, \ldots, \vec{\varphi}_{d}\right) ; \vec{\varphi}_{i} \in \mathbb{C}\{x\}^{\mu_{i}} \text { for } i=1, \ldots, d\right\} .
$$

Therefore, to complete the proof of Theorem 2 it is enough to prove

Theorem 4. Every solution $u(t, x) \in \mathcal{S}_{+}$is expressed in the form

$$
u(t, x)=U\left(\vec{\varphi}_{1}, \ldots, \vec{\varphi}_{d}\right)
$$

for some unique $\vec{\varphi}_{i} \in \mathbb{C}\{x\}^{\mu_{i}}(i=1, \ldots, d)$.

The proof of this theorem is almost the same as that of Théorème 4 of Gérard-Tahara [5]: but, for the sake of convenience of readers we will give here a refined version of the proof.

Let $a_{1}, \ldots, a_{d}$ be the ones in (3.2). Set $a_{0}=0$ and $a_{d+1}=\infty$. Our proof of Theorem 4 is based on the following proposition:

Proposition 4. Let $u_{1}(t, x) \in \mathcal{S}_{+}$and $u_{2}(t, x) \in \mathcal{S}_{+}$.

(1) We have $u_{i}(t, x)=O\left(t^{\sigma}, \widetilde{\mathcal{O}}_{+}\right)$(as $\left.t \longrightarrow 0\right)$ for $i=1,2$.

(2) If $s$ satisfies $a_{i-1}<s<a_{i}$ for some $i \in\{1, \ldots, d\}$ and if $u_{1}-u_{2}=$ $O\left(t^{s}, \widetilde{\mathcal{O}}_{+}\right)($as $t \longrightarrow 0)$ holds, we have $u_{1}-u_{2}=O\left(t^{\sigma N_{i}}, \widetilde{\mathcal{O}}_{+}\right)($as $t \longrightarrow 0)$.

(3) If $u_{1}-u_{2}=O\left(t^{\sigma N_{i}}, \widetilde{\mathcal{O}}_{+}\right)$(as $\left.t \longrightarrow 0\right)$ holds for some $i \in\{1, \ldots, d\}$, we have

$$
u_{1}-u_{2}=\sum_{j=1}^{\mu_{i}} \varphi_{i, j}(x) v_{i, j}(t, x)+O\left(t^{\sigma\left(N_{i}+1\right)}, \widetilde{\mathcal{O}}_{+}\right) \quad(\text { as } t \longrightarrow 0)
$$

for some $\varphi_{i, j}(x) \in \mathbb{C}\{x\}\left(j=1, \ldots, \mu_{i}\right)$.

(4) If $s>a_{d}$ holds and if $u_{1}-u_{2}=O\left(t^{s}, \widetilde{\mathcal{O}}_{+}\right)$(as $\left.t \longrightarrow 0\right)$, then we have $u_{1}=u_{2}$ in $\widetilde{\mathcal{O}}_{+}$.

Let us admit this proposition for a moment. By using this result we can give a proof of Theorem 4 as follows.

Proof of Theorem 4. Let $u(t, x) \in \mathcal{S}_{+}$. Set $u_{0}=U(\overrightarrow{0}, \ldots, \overrightarrow{0})$. Then, by the definition we see that $u-u_{0}=O\left(t^{s}, \widetilde{\mathcal{O}}_{+}\right)($as $t \longrightarrow 0)$ for some $a_{0}=0<s<a_{1}$. Therefore, by (2),(3) of Proposition 4 we have

$$
u-u_{0}=\sum_{j=1}^{\mu_{1}} \varphi_{1, j}(x) v_{1, j}(t, x)+O\left(t^{\sigma\left(N_{1}+1\right)}, \widetilde{\mathcal{O}}_{+}\right) \quad(\text { as } t \longrightarrow 0)
$$


for some $\varphi_{1, j}(x) \in \mathbb{C}\{x\}\left(j=1, \ldots, \mu_{1}\right)$. Using this $\vec{\varphi}_{1}=\left(\varphi_{1,1}, \ldots, \varphi_{1, \mu_{1}}\right)$ we set $u_{1}=U\left(\vec{\varphi}_{1}, \overrightarrow{0}, \ldots, \overrightarrow{0}\right)$. Then, by (8.2) and (2) of Proposition 3 we have

$$
\begin{aligned}
u-u_{1}= & \left(u-u_{0}\right)-\left(u_{1}-u_{0}\right) \\
= & \left(u-u_{0}\right)-\left(U\left(\vec{\varphi}_{1}, \overrightarrow{0}, \ldots, \overrightarrow{0}\right)-U(\overrightarrow{0}, \overrightarrow{0}, \ldots, \overrightarrow{0})\right) \\
= & {\left[\sum_{j=1}^{\mu_{1}} \varphi_{1, j}(x) v_{1, j}(t, x)+O\left(t^{\sigma\left(N_{1}+1\right)}, \widetilde{\mathcal{O}}_{+}\right)\right] } \\
& \quad-\left[\sum_{j=1}^{\mu_{1}} \varphi_{1, j}(x) v_{1, j}(t, x)+O\left(t^{\sigma\left(N_{1}+1\right)}, \widetilde{\mathcal{O}}_{+}\right)\right] \\
= & O\left(t^{\sigma\left(N_{1}+1\right)}, \widetilde{\mathcal{O}}_{+}\right)(\text {as } t \longrightarrow 0) .
\end{aligned}
$$

Since $a_{1}<\sigma\left(N_{1}+1\right)<a_{2}$ holds, by using (2),(3) of Proposition 4 again we see that $u-u_{1}$ is expressed in the form

$$
u-u_{1}=\sum_{j=1}^{\mu_{2}} \varphi_{2, j}(x) v_{2, j}(t, x)+O\left(t^{\sigma\left(N_{2}+1\right)}, \widetilde{\mathcal{O}}_{+}\right) \quad(\text { as } t \longrightarrow 0)
$$

for some $\varphi_{2, j}(x) \in \mathbb{C}\{x\}\left(j=1, \ldots, \mu_{2}\right)$. Using this $\vec{\varphi}_{2}=\left(\varphi_{2,1}, \ldots, \varphi_{2, \mu_{2}}\right)$ we set $u_{2}=U\left(\vec{\varphi}_{1}, \vec{\varphi}_{2}, \overrightarrow{0}, \ldots, \overrightarrow{0}\right)$. Then, by (8.3) and (2) of Proposition 3 we have

$$
\begin{aligned}
u-u_{2}= & \left(u-u_{1}\right)-\left(u_{2}-u_{1}\right) \\
= & \left(u-u_{1}\right)-\left(U\left(\vec{\varphi}_{1}, \vec{\varphi}_{2}, \overrightarrow{0}, \ldots, \overrightarrow{0}\right)-U\left(\vec{\varphi}_{1}, \overrightarrow{0}, \overrightarrow{0}, \ldots, \overrightarrow{0}\right)\right) \\
= & {\left[\sum_{j=1}^{\mu_{1}} \varphi_{2, j}(x) v_{2, j}(t, x)+O\left(t^{\sigma\left(N_{2}+1\right)}, \widetilde{\mathcal{O}}_{+}\right)\right] } \\
& \quad-\left[\sum_{j=1}^{\mu_{1}} \varphi_{2, j}(x) v_{2, j}(t, x)+O\left(t^{\sigma\left(N_{2}+1\right)}, \widetilde{\mathcal{O}}_{+}\right)\right] \\
= & O\left(t^{\sigma\left(N_{2}+1\right)}, \widetilde{\mathcal{O}}_{+}\right)(\text {as } t \longrightarrow 0) .
\end{aligned}
$$

Repeating the same argument as above we can find $\vec{\varphi}_{i}=\left(\varphi_{i, 1}, \ldots, \varphi_{i, \mu_{i}}\right) \in$ $\mathbb{C}\{x\}^{\mu_{i}}(i=1, \ldots, d)$ so that if we set

$$
u_{p}=U\left(\vec{\varphi}_{1}, \ldots, \vec{\varphi}_{p}, \overrightarrow{0}, \ldots, \overrightarrow{0}\right), \quad p=1, \ldots, d
$$

we have the asymptotics

$$
u-u_{p}=O\left(t^{\sigma\left(N_{p}+1\right)}, \widetilde{\mathcal{O}}_{+}\right) \quad(\text { as } t \longrightarrow 0), \quad p=1, \ldots, d .
$$

Thus, if we take the case $p=d$ we have $u-u_{d}=O\left(t^{\sigma\left(N_{d}+1\right)}, \widetilde{\mathcal{O}}_{+}\right)$(as $t \longrightarrow 0)$ : then, by the condition $\sigma\left(N_{d}+1\right)>a_{d}$ and by (4) of Proposition 4 we obtain the result $u=u_{d}$ in $\widetilde{\mathcal{O}}_{+}$. 
This proves that $u(t, x) \in \mathcal{S}_{+}$is expressed in the form (8.1) for some $\vec{\varphi}_{i}(x) \in \mathbb{C}\{x\}^{\mu_{i}}(i=1, \ldots, d)$. The uniqueness of $\vec{\varphi}_{i}(x)(i=1, \ldots, d)$ follows from (3) of Proposition 3.

Now, let us give a proof of Proposition 4. We note:

Lemma 10. Assume that $w(t, x) \in \widetilde{\mathcal{O}}_{+}$and $f(t, x) \in \widetilde{\mathcal{O}}_{+}$satisfy the equation

$$
C\left(t \frac{\partial}{\partial t}, x\right) w=f(t, x)
$$

(1) If $a_{i-1}<s<b<a_{i}$ holds for some $i \in\{1, \ldots, d\}$, if $w(t, x)=$ $O\left(t^{s}, \widetilde{\mathcal{O}}_{+}\right)($as $t \longrightarrow 0)$ and if $f(t, x)=O\left(t^{b}, \widetilde{\mathcal{O}}_{+}\right)($as $t \longrightarrow 0)$, then we have $w(t, x)=O\left(t^{b}, \widetilde{\mathcal{O}}_{+}\right)($as $t \longrightarrow 0)$.

(2) If $a_{i-1}<s<a_{i}<b<a_{i+1}$ holds for some $i \in\{1, \ldots, d\}$, if $w(t, x)=$ $O\left(t^{s}, \widetilde{\mathcal{O}}_{+}\right)($as $t \longrightarrow 0)$ and if $f(t, x)=O\left(t^{b}, \widetilde{\mathcal{O}}_{+}\right)$(as $\left.t \longrightarrow 0\right)$, then we have

$$
w(t, x)=\sum_{j=1}^{\mu_{i}} \varphi_{i, j}(x) v_{i, j}(t, x)+O\left(t^{b}, \widetilde{\mathcal{O}}_{+}\right) \quad(\text { as } t \longrightarrow 0)
$$

for some $\varphi_{i, j}(x) \in \mathbb{C}\{x\}\left(j=1, \ldots, \mu_{i}\right)$.

Proof. Set

$$
P_{1}=\prod_{k=0}^{i-1} C_{k}\left(t \frac{\partial}{\partial t}, x\right), \quad P_{2}=\prod_{k=i}^{d} C_{k}\left(t \frac{\partial}{\partial t}, x\right),
$$

and $w_{2}=P_{1} w$. Then we have $C(t \partial / \partial t, x)=P_{2} P_{1}$ and $P_{2} w_{2}=f$. Since $f(t, x)=O\left(t^{b}, \widetilde{\mathcal{O}}_{+}\right)($as $t \longrightarrow 0)$ and since $b<a_{k}$ holds for all $k=i, \ldots, d$, by applying (3) of Lemma 4 to the equation $P_{2} w_{2}=f$ we have $w_{2}(t, x)=$ $O\left(t^{b}, \widetilde{\mathcal{O}}_{+}\right)($as $t \longrightarrow 0)$. Since $w(t, x)=O\left(t^{s}, \widetilde{\mathcal{O}}_{+}\right)($as $t \longrightarrow 0)$ and since $a_{k}<s$ holds for all $k=0, \ldots, i-1$, by applying (2) of Lemma 4 to the equation $P_{1} w=w_{2}$ we obtain $w(t, x)=O\left(t^{b}, \widetilde{\mathcal{O}}_{+}\right)$(as $\left.t \longrightarrow 0\right)$. This proves the part (1).

Next let us prove (2). Assume that $a_{i-1}<s<a_{i}<b<a_{i+1}$ holds for some $i \in\{1, \ldots, d\}$. Set

$$
P_{3}=\prod_{k=i+1}^{d} C_{k}\left(t \frac{\partial}{\partial t}, x\right) \quad \text { if } i<d,
$$

and $P_{3}=i d e n t i t y$ if $i=d$. Set also $w_{1}=C_{i}(t \partial / \partial t, x) w$ and $w_{3}=P_{1} w_{1}$. Then we have $C(t \partial / \partial t, x)=P_{3} P_{1} C_{i}$ and $P_{3} w_{3}=f$. Since $a_{i-1}<s<a_{i}<b<a_{i+1}$ 
holds, by the same argument as above we see that $w_{3}(t, x)=O\left(t^{b}, \widetilde{\mathcal{O}}_{+}\right)$(as $t \longrightarrow 0)$ and $w_{1}(t, x)=O\left(t^{b}, \widetilde{\mathcal{O}}_{+}\right)($as $t \longrightarrow 0)$.

Now let us consider the relation $C_{i} w=w_{1}$. We already know that $w_{1}(t, x)=O\left(t^{b}, \widetilde{\mathcal{O}}_{+}\right)$(as $\left.t \longrightarrow 0\right)$ and $s<a_{i}<b$ hold. Hence, by (1) of Lemma 4 we have a unique solution $W(t, x)$ of $C_{i} W=w_{1}$ satisfying $W=O\left(t^{b}, \widetilde{\mathcal{O}}_{+}\right)$(as $t \longrightarrow 0)$. Then, we have $C_{i}(w-W)=0$ and therefore by using the fundamental solutions $\left\{v_{i, 1}(t, x), \ldots, v_{i, \mu_{i}}(t, x)\right\}$ we can express $(w-W)(t, x)$ in the form

$$
w-W=\sum_{j=1}^{\mu_{i}} \varphi_{i, j}(x) v_{i, j}(t, x)
$$

for some $\varphi_{i, j}(x) \in \mathbb{C}\{x\}\left(j=1, \ldots, \mu_{i}\right)$. This leads us to the conclusion of the part (2).

Proof of Proposition 4. First we will prove (1) only in the case $i=1$. Note that our equation for $u_{1}$ is written in the form

$$
C\left(t \frac{\partial}{\partial t}, x\right) u_{1}=H\left[u_{1}\right]
$$

where

$$
H\left[u_{1}\right]=b(x) t+\sum_{p+|q| \geq 2} b_{p, q}(x) t^{p} \prod_{(j, \alpha) \in I_{m}}\left[\mathcal{D}_{j, \alpha} u_{1}\right]^{q_{j, \alpha}} .
$$

It is easy to see that the operator $H[f]$ with $f \in \widetilde{\mathcal{O}}_{+}$satisfies the following properties: $\gamma-1) H[\cdot]$ is a mapping from $\widetilde{\mathcal{O}}_{+}$to $\widetilde{\mathcal{O}}_{+}$, and $\left.\gamma-2\right)$ if $f=O\left(t^{\varepsilon}, \widetilde{\mathcal{O}}_{+}\right)$ (as $t \longrightarrow 0$ ) for some $\varepsilon>0$ we have $H[f]=O\left(t^{r}, \widetilde{\mathcal{O}}_{+}\right.$) (as $t \longrightarrow 0$ ) for any $r<\min \{1,2 \varepsilon\}$.

Let $u_{1}(t, x) \in \mathcal{S}_{+}$. By the definition we have $u_{1}(t, x)=O\left(t^{s}, \widetilde{\mathcal{O}}_{+}\right)$(as $t \longrightarrow 0$ ) for some $s>0$. If $s \geq \sigma$ we have nothing to prove. If $s<\sigma$ we choose a sequence $s_{0}, s_{1}, \ldots, s_{p}$ such that $s_{0}=s<s_{1}<\cdots<s_{p}=\sigma(<1)$ and that $s_{k}<\min \left\{1,2 s_{k-1}\right\}$ holds for $k=1, \ldots, p$; then we can prove the property $u_{1}(t, x)=O\left(t^{\sigma}, \widetilde{\mathcal{O}}_{+}\right)($as $t \longrightarrow 0)$ in the following way.

Note that $a_{0}=0<s_{k}<a_{1}$ for $k=0,1, \ldots, p$ and that $u_{1}=O\left(t^{s_{0}}, \widetilde{\mathcal{O}}_{+}\right)$ (as $t \longrightarrow 0$ ) is known. Therefore, by $\gamma-2)$ we have $H\left[u_{1}\right]=O\left(t^{s_{1}}, \widetilde{\mathcal{O}}_{+}\right)$(as $t \longrightarrow 0)$ and by applying (1) of Lemma 10 to (8.5) we have $u_{1}=O\left(t^{s_{1}}, \widetilde{\mathcal{O}}_{+}\right)$ (as $t \longrightarrow 0$ ). Then, by $\gamma-2$ ) we have $H\left[u_{1}\right]=O\left(t^{s_{2}}, \widetilde{\mathcal{O}}_{+}\right)($as $t \longrightarrow 0$ ) and by applying (1) of Lemma 10 to (8.5) again we obtain $u_{1}=O\left(t^{s_{2}}, \widetilde{\mathcal{O}}_{+}\right)$(as $t \longrightarrow 0)$. Thus, by repeating the same argument we obtain $u_{1}=O\left(t^{s_{p}}, \widetilde{\mathcal{O}}_{+}\right)$ (as $t \longrightarrow 0$ ). Since $s_{p}=\sigma$, this completes the proof of the part (1). 
Next let us show (2). By (1) we have $u_{i}(t, x)=O\left(t^{\sigma}, \widetilde{\mathcal{O}}_{+}\right)$(as $\left.t \longrightarrow 0\right)$ for $i=1,2$. Set $w=u_{1}-u_{2}$. Then we have $w(t, x)=O\left(t^{\sigma}, \widetilde{\mathcal{O}}_{+}\right)$(as $\left.t \longrightarrow 0\right)$ and we see that $w(t, x)$ satisfies the equation

$$
C\left(t \frac{\partial}{\partial t}, x\right) w=G[w]
$$

where

$$
\begin{aligned}
G[w]=\sum_{p+|q| \geq 2,|q| \geq 1} b_{p, q}(x) t^{p}\left[\prod_{(j, \alpha) \in I_{m}}\left(\mathcal{D}_{j, \alpha} w+\mathcal{D}_{j, \alpha} u_{2}(t, x)\right)^{q_{j, \alpha}}\right. & \\
& \left.-\prod_{(j, \alpha) \in I_{m}}\left(\mathcal{D}_{j, \alpha} u_{2}(t, x)\right)^{q_{j, \alpha}}\right] .
\end{aligned}
$$

We see easily that $G[\cdot]$ is a mapping from $\widetilde{\mathcal{O}}_{+}$to $\widetilde{\mathcal{O}}_{+}$and that if $f=O\left(t^{\varepsilon}, \widetilde{\mathcal{O}}_{+}\right)$ (as $t \longrightarrow 0$ ) for some $\varepsilon>0$ we have $G[f]=O\left(t^{r}, \widetilde{\mathcal{O}}_{+}\right.$) (as $t \longrightarrow 0$ ) for any $r \leq \min \{\sigma+\varepsilon, 2 \varepsilon\}$.

Assume that $a_{i-1}<s<a_{i}$ holds for some $i \in\{1, \ldots, d\}$ and that $w=$ $u_{1}-u_{2}=O\left(t^{s}, \widetilde{\mathcal{O}}_{+}\right)$(as $t \longrightarrow 0$ ). If $s \geq \sigma N_{i}$ we have nothing to prove in (2). If $s<\sigma N_{i}$ we choose a sequence $s_{0}, s_{1}, \ldots, s_{p}$ such that $s_{0}=s<s_{1}<$ $\cdots<s_{p}=\sigma N_{i}$ and that $s_{k} \leq \min \left\{\sigma+s_{k-1}, 2 s_{k-1}\right\}$ holds for $k=1, \ldots, p$; then, applying the same argument as in the proof of (1) to the equation (8.6) we obtain $w(t, x)=O\left(t^{s_{p}}, \widetilde{\mathcal{O}}_{+}\right)$(as $\left.t \longrightarrow 0\right)$. This proves the part $(2)$.

The proof of (3) is as follows. Assume that $w=u_{1}-u_{2}=O\left(t^{\sigma N_{i}}, \widetilde{\mathcal{O}}_{+}\right)$ (as $t \longrightarrow 0)$. Then we have $G[w]=O\left(t^{\sigma\left(N_{i}+1\right)}, \widetilde{\mathcal{O}}_{+}\right)($as $t \longrightarrow 0)$ and therefore by applying (2) of Lemma 10 to the equation (8.6) we obtain the conclusion of the part (3).

Lastly, we note that the part (4) is the same as Proposition 3 of GérardTahara [5] and so we omit the proof. We remark that the part (4) can be proved also by the same argument as in Tahara [13],[14].

Remark 3. Let $\left\{v_{i, 1}(t, x), \ldots, v_{i, \mu_{i}}(t, x)\right\}$ be a fundamental system of $\widetilde{\mathcal{O}}_{+}$-solutions of $(3.6)_{i}$ as before. The following assertion will be easily verified: the system $\left\{v_{i, j}(t, x) ; 1 \leq i \leq d, 1 \leq j \leq \mu_{i}\right\}$ is a fundamental system of $\widetilde{\mathcal{O}}_{+}$-solutions of the equation $C(t \partial / \partial t, x) v=0$. See the condition (1) in Theorem 2. 


\section{References}

[1] Baouendi, M. S. and Goulaouic, G., Cauchy problem with characteristic initial hypersurface, Comm. Pure Appl. Math., 26 (1973), 455-475.

[2] Chen, H. and Tahara, H., On totally characteristic type non-linear partial differential equations in the complex domain, Publ. RIMS, Kyoto Univ., 35 (1999), 621-636.

[3] , On the holomorphic solution of non-linear totally characteristic equations, Math. Nachr., 219 (2000), 85-96.

[4] Gérard, R. and Tahara, H., Holomorphic and singular solutions of nonlinear singular first order partial differential equations, Publ. RIMS, Kyoto Univ., 26 (1990), 979-1000.

[5] - Solutions holomorphes et singulières d'équations aux dérivées partielles singulières non linéaires, Publ. RIMS, Kyoto Univ., 29 (1993), 121-151.

[6] _ Singular nonlinear partial differential equations, Vieweg, 1996.

[7] - Holomorphic and singular solutions of non-linear singular partial differential equations, II. "Structure of differential equations, Katata/Kyoto, 1995 " (edited by M. Morimoto and T. Kawai), 135-150, World Scientific, 1996.

[8] Hörmander, L., Linear partial differential operators, Springer, 1963.

[9] Kobayashi, T., Singular solutions and prolongation of holomorphic solutions to nonlinear differential equations, Publ. RIMS. Kyoto Univ., 34 (1998), 43-63.

[10] Mandai, T., The method of Frobenius to Fuchsian partial differential equations, J. Math. Soc. Japan, 52 (2000), 645-672.

[11] Nagumo, M., Über das Anfangswertproblem Parteller Differentialgleichungen, Japan. J. Math., 18 (1941), 41-47.

[12] Lope, J. E. C. and Tahara, H., On the analytic continuation of solutions to nonlinear partial differential equations, J. Math. Pures Appl., 81 (2002), 811-826.

[13] Tahara, H., Uniqueness of the solution of non-linear singular partial differential equations, J. Math. Soc. Japan, 48 (1996), 729-744.

[14] On the uniqueness theorem for nonlinear singular partial differential equations, J. Math. Sci. Univ. Tokyo, 5 (1998), 477-506.

[15] _ On the singularities of solutions of nonlinear partial differential equations in the complex domain, "Microlocal analysis and complex Fourier analysis" (edited by T. Kawai and K. Fujita), 273-283, World Scientific, 2002.

[16] Solvability of nonlinear totally characteristic type partial differential equations with resonances, J. Math. Soc. Japan, 55 (2003), 1095-1113.

[17] Yamazawa, H., Singular solutions of the Briot-Bouquet type partial differential equations, J. Math. Soc. Japan, 55 (2003). 